\title{
Space-time adaptive ADER discontinuous Galerkin schemes for nonlinear hyperelasticity with material failure
}

\author{
Maurizio Tavelli ${ }^{\mathrm{a}}$, Simone Chiocchetti ${ }^{\mathrm{a}}$, Evgeniy Romenski $^{\mathrm{a}, \mathrm{b}}$, Alice-Agnes Gabriel ${ }^{\mathrm{c}}$, Michael Dumbser $^{* a}$ \\ ${ }^{a}$ Laboratory of Applied Mathematics, University of Trento, Via Mesiano 77, 38123 Trento, Italy \\ ${ }^{b}$ Sobolev Institute of Mathematics, 4 Acad. Koptyug Avenue, 630090 Novosibirsk, Russia \\ ${ }^{c}$ Ludwig-Maximilians Universität München (LMU), Theresienstr. 41, 80333 München, Germany
}

\begin{abstract}
We are concerned with the numerical solution of a unified first order hyperbolic formulation of continuum mechanics that goes back to the work of Godunov, Peshkov and Romenski [64, 67, 96] (GPR model) and which is an extension of nonlinear hyperelasticity that is able to describe simultaneously nonlinear elasto-plastic solids at large strain, as well as viscous and ideal fluids. The proposed governing PDE system also contains the effect of heat conduction and can be shown to be symmetric and thermodynamically compatible, as it obeys the first and second law of thermodynamics.

In this paper we extend the GPR model to the simulation of nonlinear dynamic rupture processes, which can be achieved by adding an additional scalar to the governing PDE system. This extra parameter describes the material damage and is governed by an advection-reaction equation, where the stiff and highly nonlinear reaction mechanisms depend on the ratio of the local equivalent stress to the yield stress of the material. The stiff reaction mechanisms are integrated in time via an efficient exponential time integrator. Due to the multiple spatial and temporal scales involved in the problem of crack generation and propagation, the model is solved on space-time adaptive Cartesian meshes using high order accurate discontinuous Galerkin finite element schemes endowed with an a posteriori subcell finite volume limiter.

A key feature of our new model is the use of a twofold diffuse interface approach that allows the cracks to form anywhere and at any time, independently of the chosen computational grid, which is simply adaptive Cartesian (AMR). This is substantially different from many fracture modeling approaches that need to resolve discontinuities explicitly, such as for example dynamic shear rupture models used in computational seismology, where the geometry of the rupture fault needs to prescribed a priori. We furthermore make use of a scalar volume fraction function $\alpha$ that indicates whether a given spatial point is inside the solid $(\alpha=1)$ or outside $(\alpha=0)$, thus allowing the description of solids of arbitrarily complex shape.

We show extensive numerical comparisons with experimental results for stress-strain diagrams of different real materials and for the generation and propagation of fracture in rocks and pyrex glass at low and high velocities. Overall, a very good agreement between numerical simulations and experiments is obtained. The proposed model is also naturally able to describe material fatigue.
\end{abstract}

Keywords: arbitrary high-order Discontinuous Galerkin schemes, a posteriori subcell finite volume limiter, exponential time integrator for stiff source terms, unified first order hyperbolic formulation of nonlinear continuum mechanics, crack generation and dynamic rupture, material fatigue 


\section{Introduction}

Nonlinear solid mechanics with large deformations and material failure is of fundamental importance in mechanical and civil engineering for the computation and design of structures under dynamic loads. Particularly relevant for engineering applications are crack formation in intact materials and the phenomenon of material fatigue. Also in geophysics nonlinear large deformation solid mechanics is relevant for the description of dynamic rupture processes in earthquakes. One major difficulty in numerical modeling of crack propagation is the introduction of strong discontinuities in the displacement field in the vicinity of the crack. Numerical methods which require the discontinuities to be exactly resolved by the mesh generally require the geometry of the fractures to be predefined and thus typically only permit small deformations. Alternative methods which allow representing discontinuities at the sub-element level, such as the eXtended Finite Element Method (XFEM) [87], introduce singularities when an interface intersects a cell vertex, and can become difficult to implement in an efficient manner in $3 \mathrm{D}$.

While solid mechanics is usually formulated more naturally in Lagrangian coordinates, it was shown in [65] that a mathematical formulation of solid mechanics is also possible in Eulerian coordinates. This formulation was put in the more general mathematical framework of symmetric hyperbolic and thermodynamically compatible (SHTC) systems in Godunov-type form [62] in a series of papers $[63,106,66,67]$. Similar hyperelastic models were also used later in $[13,14,10$, $96,47,36,1,77,76]$, including strain hardening and plastic deformations. In order to model complex geometries, diffuse interface methods can be employed, where a scalar volume fraction represents the occupation of each control volume by the solid material. The most important contributions concerning diffuse interface models for the description of compressible hyperelastic multi-material flows have been made in a series of papers by Favrie and Gavrilyuk and collaborators, see $[60,56,55,90,91,12]$.

The main objectives of this paper are to show that nonlinear elasto-plastic material behaviour under large strains with material damage can be described via nonlinear hyperelasticity [65] and the framework of SHTC systems in Godunov form [62, 106, 97] and that the resulting governing PDE system can be robustly solved with high order accurate discontinuous Galerkin (DG) finite element schemes with a posteriori subcell finite volume limiter, see [52].

Currently the generally accepted model of continual failure and rupture is the phase-field model, which was proposed by Francfort and Marigo in [57] for brittle materials and served as the basis for further theoretical and numerical developments. The phase-field model consists in representing the crack as a diffuse interface between intact zones of the medium using the order parameter field and is usually formulated as a minimization of the energy functional and is solved by the finite element method. Numerous generalizations of the model [57] for the brittle and ductile fracture and the fracture of porous material have been developed and their description can be found, for example, in $[6,7,30,25,112]$ and references therein. Note that all phase-field models are well suited for quasi-static problems, and the corresponding partial differential equations contain second-order (parabolic) terms, which is different from the first order hyperbolic PDE system proposed in this paper that allows to construct a rate-dependent and fully dynamic model of material failure, including also material fatigue.

Numerical modeling of secondary cracks during dynamic fracture propagation is also an important problem to consider in earthquake physics [79]: high stress concentrations at the earthquake

* Corresponding author

Email addresses: m.tavelli@unitn.it (Maurizio Tavelli), simone.chiocchetti@unitn.it (Simone

Chiocchetti), evrom@math.nsc.ru (Evgeniy Romenski), gabriel@geophysik.uni-muenchen.de

(Alice-Agnes Gabriel), michael.dumbser@unitn.it (Michael Dumbser*) 
rupture tip feedback with off-fault anelastic processes such as continuum damage $[84,20,117,116]$, explicit tensile and shear fracturing [128, 35, 92, 93] or off-fault plasticity $[8,58]$.

In this paper we first present an extended diffuse interface version of the Godunov-PeshkovRomenski (GPR) hyperbolic model of continuum mechanics [96, 47, 125, 95] that includes an additional evolution equation for a scalar material damage variable $\xi$, where $\xi=0$ indicates the fully intact material, while $\xi=1$ represents a fully damaged material. The dynamics of the material damage is driven by a reaction-type source term (similar to reaction source terms in chemistry), that depends on the ratio of equivalent stress to yield stress. This idea goes back to [103, 107, 102], where the dynamics of failure waves was discussed in the context of hyperelasticity. To account for nonlinear elasto-plastic materials, the GPR model includes a strain relaxation mechanism, see [96, 47]. We also suggest some simple mixture rules for the computation of the Lamé parameters and the strain relaxation time scale of the material as a function of the damage variable $\xi$. All above gives us a highly flexible model that allows us to simulate large deformations with rate- and temperaturedependent failure of brittle and ductile materials. Note that an anisotropic thermodynamically consistent damage model based on the hyperelastic Godunov-Romenski approach has already been proposed in [11]. In [11] a damage deformation gradient was introduced in addition to an elastic and a plastic deformation gradient.

The numerical methods employed in this paper essentially rely on the framework of Godunovtype finite volume schemes, see e.g. [61, 70, 54, 123, 105, 127, 69, 119, 124, 122], which are then used as a posteriori subcell limiter $[52,110,111]$ of a high order discontinuous Galerkin finite element method [33, 32, 31, 34] on space-time adaptive Cartesian AMR meshes [19, 17, 16, 15, 18, 132]. The concept of a posteriori limiting was introduced in a series of papers by Clain and Loubère and coworkers in $[29,37,38,81]$ and relies on the a posteriori detection of troubled cells and subsequent local order reduction of the scheme, in order to ensure essentially non-oscillatory behaviour and positivity of the numerical solution. For more details, the reader is referred to the above references. To the best knowledge of the authors, this is the first time that high order discontinuous Galerkin finite element schemes are applied to a nonlinear hyperelastic model of elasto-plastic media with material failure and diffuse interface representation of the geometry of the medium.

The rest of this paper is organized as follows. In Section 2 we introduce and discuss the underlying mathematical model. In Section 3 we present the numerical method employed in this paper to solve the governing partial differential equations. Our approach is based on high order discontinuous Galerkin (DG) finite element schemes with a posteriori subcell finite volume limiting on space-time adaptive meshes (AMR). In order to deal with the very stiff reaction source terms in the evolution equation of the damage variable, an accurate and efficient ODE solver based on exponential time integration is presented. The time integration method is validated for different material behaviours (brittle, ductile and fatigued materials) against standard solvers like LSODA. In Section 4 we present computational results for a set of different test cases containing crack generation, including also comparisons with experimental data for some of the test problems. The paper closes with Section 5, where concluding remarks and an outlook to future research are given. 


\section{Mathematical model}

\subsection{Governing partial differential equations}

A diffuse interface formulation for moving nonlinear solids of arbitrary geometry and at large strain is given by the following PDE system in Eulerian coordinates:

$$
\begin{aligned}
& \frac{\partial \alpha}{\partial t}+v_{k} \cdot \frac{\partial \alpha}{\partial x_{k}}=0 \\
& \frac{\partial \rho}{\partial t}+\frac{\partial\left(\rho v_{k}\right)}{\partial x_{k}}=0 \\
& \frac{\partial\left(\alpha \rho v_{i}\right)}{\partial t}+\frac{\partial\left(\alpha \rho v_{i} v_{k}+\alpha p \delta_{i k}-\alpha \sigma_{i k}\right)}{\partial x_{k}}=0 \\
& \frac{\partial A_{i k}}{\partial t}+\frac{\partial A_{i m} v_{m}}{\partial x_{k}}+v_{m}\left(\frac{\partial A_{i k}}{\partial x_{m}}-\frac{\partial A_{i m}}{\partial x_{k}}\right)=-\frac{1}{\theta_{1}\left(\tau_{1}\right)} E_{A_{i k}}, \\
& \frac{\partial J_{k}}{\partial t}+\frac{\partial\left(v_{m} J_{m}+T\right)}{\partial x_{k}}+v_{m}\left(\frac{\partial J_{k}}{\partial x_{m}}-\frac{\partial J_{m}}{\partial x_{k}}\right)=-\frac{1}{\theta_{2}\left(\tau_{2}\right)} E_{J_{k}}, \\
& \frac{\partial \xi}{\partial t}+v_{k} \cdot \frac{\partial \xi}{\partial x_{k}}=-\theta E_{\xi}, \quad \\
& \frac{\partial \rho S}{\partial t}+\frac{\partial\left(\rho S v_{k}+\rho E_{J_{k}}\right)}{\partial x_{k}}=\frac{\rho}{T}\left(\frac{1}{\theta_{1}} E_{A_{i k}} E_{A_{i k}}+\frac{1}{\theta_{2}} E_{J_{k}} E_{J_{k}}+\theta E_{\xi} E_{\xi}\right) \geq 0 \\
& \frac{\partial \rho E}{\partial t}+\frac{\partial\left(v_{k} \rho E+v_{i}\left(p \delta_{i k}-\sigma_{i k}\right)\right)}{\partial x_{k}}=0 .
\end{aligned}
$$

Throughout this paper we make use of the Einstein summation convention over repeated indices. Here (1a) is the evolution equation for the colour function $\alpha$ that is needed in the diffuse interface approach (DIM) as introduced in $[115,24]$ for the description of solids of arbitrary geometry; (1b) is the mass conservation law and $\rho$ is the material density; $(1 \mathrm{c})$ is the momentum conservation law and $v_{i}$ is the velocity field; (1d) is the evolution equation for the distortion field $\mathbf{A}=A_{i k}$ (basis triad ${ }^{1}$ ); (1e) is the evolution equation for the specific thermal impulse $J_{k}$ constituting the heat conduction in the medium via a hyperbolic (non Fourier-type) model; (1f) is the evolution equation for the material damage variable $\xi \in[0,1]$, where $\xi=0$ indicates fully intact material and $\xi=1$ fully damaged material. Finally, $(1 \mathrm{~g})$ is the entropy inequality and (1h) is the energy conservation law. Other thermodynamic parameters are defined via the total energy potential $E=E(\rho, S, \mathbf{v}, \boldsymbol{A}, \mathbf{J}, \xi)$ : $\boldsymbol{\Sigma}=\Sigma_{i k}=-p \delta_{i k}+\sigma_{i k}$ is the total stress tensor $\left(\delta_{i k}\right.$ is the Kronecker delta); $p=\rho^{2} E_{\rho}$ is the contribution to the stress tensor due to volume deformations; $\sigma_{i k}=-\rho A_{j i} E_{A_{j k}}+\rho J_{i} E_{J_{k}}$ is the contribution to the stress tensor due to shear and thermal stress, and $T=E_{S}$ is the temperature. Note that in system (1) we only use a simplified diffuse interface approach, which completely neglects the dynamics of the air surrounding the solid medium. In our model the solid volume fraction $\alpha$ is only used to locate and track the shape of the moving solid, see [115]. In comparison, in the work of Favrie and Gavrilyuk and collaborators [56, 55, 91], real multi-phase flows of compressible solids embedded in compressible fluids were considered.

The dissipation in the medium includes two relaxation processes: the strain relaxation (or shear stress relaxation) characterized by the scalar function $\theta_{1}\left(\tau_{1}\right)>0$ depending on the relaxation time $\tau_{1}$ and the heat flux relaxation characterized by $\theta_{2}\left(\tau_{2}\right)>0$, depending on the relaxation time $\tau_{2}$.

Further to the above evolution equations we need to add the governing PDE for the local Lamé parameters $\lambda$ and $\mu$, material yield stress $Y_{0}$ and other material constants. For small deformations

\footnotetext{
${ }^{1}$ Note that $A_{i k}$ transforms as a set of three vectors and not as a rank two tensor under coordinate transformations. In the absence of strain relaxation source terms $\left(\tau_{1} \rightarrow \infty\right) A_{i k}$ is the deformation gradient of the material.
} 
it is sufficient to assume them as constant in time, but for large deformations those parameters have to move with the local velocity field. The resulting PDEs for the material parameters therefore read:

$$
\frac{\partial \lambda}{\partial t}+v_{k} \cdot \frac{\partial \lambda}{\partial x_{k}}=0, \quad \frac{\partial \mu}{\partial t}+v_{k} \cdot \frac{\partial \mu}{\partial x_{k}}=0, \quad \frac{\partial Y_{0}}{\partial t}+v_{k} \cdot \frac{\partial Y_{0}}{\partial x_{k}}=0 .
$$

If $\alpha=1$, then it can be immediately seen that the GPR model (1) is thermodynamically compatible as soon as the total energy is defined as a function of parameters of state [106, 67, 96, 47], since it satisfies the first and second principle of thermodynamics, see (1h) and (1g), which are the total energy conservation law and the entropy inequality, respectively. Note that system (1) is overdetermined, that is the number of equations is one more than the number of unknown parameters of state. In fact, as is always the case for SHTC systems, the energy equation (1h) is a consequence of all other equations and we underline that in the numerical test cases presented in this paper, we solve the energy conservation law (1h) instead of the entropy inequality ( $1 \mathrm{~g})$.

Furthermore, in order to close the system one must specify the total energy potential as a function of the other state variables, i.e. $E=E(\rho, S, \mathbf{v}, \boldsymbol{A}, \mathbf{J}, \xi)$. This potential then generates all terms in the fluxes and source terms by means of its partial derivatives with respect to the state variables. Therefore, as already discussed in [47], the energy specification is the key step in the model formulation.

Here, we make the choice $E=E_{1}+E_{2}+E_{3}$, decomposing the energy into a contribution from the microscale $E_{1}$, the mesoscale $E_{2}$ and the macroscale $E_{3}$. The individual contributions read as follows:

$$
E_{1}=\frac{K}{2 \rho_{0}}\left(1-\frac{\rho}{\rho_{0}}\right)^{2}+c_{v} T_{0}\left(\frac{\rho}{\rho_{0}}\right)\left(e^{S / c_{v}}-1\right),
$$

which is the equation of state (EOS) of the medium, where $\rho_{0}$ is the reference mass density and $K=\lambda+\frac{2}{3} \mu$ is the bulk modulus expressed in terms of the two Lamé parameters $\lambda$ and $\mu$, which depend on the damage variable $\xi, c_{v}$ is the heat capacity at constant volume and $T_{0}$ is a reference temperature. The mesoscale energy in our model reads as

$$
E_{2}=\frac{1}{4} c_{s}^{2} \stackrel{\circ}{G}_{i j} \stackrel{\circ}{G}_{i j}+\frac{1}{2} c_{h}^{2} J_{i} J_{i}
$$

where $c_{s}=\sqrt{\frac{\mu}{\rho_{0}}}$ is the shear sound speed and $c_{h}$ is related to the speed of heat waves in the medium (also called the second sound [98], or the speed of a phonon). For an alternative choice of $E_{2}$ with better mathematical properties, see [90]. Here $\stackrel{\circ}{\mathbf{G}}=\mathbf{G}-\frac{1}{3} \operatorname{tr}(\mathbf{G}) \mathbf{I}$ is the deviator of the metric (or Finger) tensor $\mathbf{G}=\mathbf{A}^{T} \mathbf{A}$ that describes the deformation of the medium. In tensor notation it reads as

$$
G_{i k}=A_{j i} A_{j k}, \quad \stackrel{\circ}{G}_{i k}=G_{i k}-\frac{1}{3} G_{j j} \delta_{i k} .
$$

Furthermore, the macro-scale energy is the classical kinetic energy and reads as

$$
E_{3}=\frac{1}{2} v_{i} v_{i}
$$

The determinant of $\mathbf{A}$ has to satisfy the algebraic constraint

$$
|\mathbf{A}|=\frac{\rho}{\rho_{0}} .
$$

Note that we define the energies $E_{1}, E_{2}$ by $(3),(4)$ in order to be able to reduce $E_{1}+E_{2}$ in case of small deformations to the quadratic energy of small strain corresponding to Hooke's law and indeed a more complex nonlinear dependence of $E_{1}, E_{2}$ on density and strain is allowed. 


\subsection{Constitutive relations for the damaged medium}

In order to make the model (1) applicable to the description of the damage processes, it is necessary to define accordingly its material parameters and constitutive relations. Our approach is based on the mixture model proposed in [103] for the damage of an elastoplastic continuum in case of small deformations and generalized later for the study of the structure of failure waves in the case of finite deformations in [107]. The idea of the mentioned approach is to consider a damaged material as a mixture of the intact and "fully damaged" materials. These two materials have their own material parameters and closing relations, such as functions characterizing the rate of shear stress relaxation. The transition from the intact material to the fully damaged material is governed by the damage variable $\xi \in[0,1]$ satisfying the kinetic equation (1f) with source term depending on the state parameters of the medium (pressure, stress and temperature). Then, if to assume that in the case of small deformations the mixture parameters of state satisfy the simple mixture rules such as an additivity of small strain and continuity of stress field, one can derive the governing equations for the damaged medium considered as an elasto-plastic continuum with material parameters and closing relations depending on the damage variable $\xi$. This mixture model of damaged medium allows one to describe a degradation of elastic moduli during the damage process and to fit experimental stress-strain diagrams depending on the strain rate.

Let us assume that the elastic moduli (Lamé constants) of the intact material $\lambda_{I}, \mu_{I}$ and of the fully damaged material $\lambda_{D}, \mu_{D}$ are known. Further, assume that the material parameters of both phases, corresponding to the heat transfer processes, such as heat capacity, thermal expansion coefficient and thermal conductivity coefficients are close to each other. The latter assumptions allow us to avoid an excessive complexity of the model.

Thus, following [103], one can define the elastic moduli in the equation of state of the damaged material as

$$
\lambda(\xi)=\frac{K_{I} K_{D}}{\tilde{K}}-\frac{2}{3} \frac{\mu_{I} \mu_{D}}{\tilde{\mu}}, \quad \mu(\xi)=\frac{\mu_{I} \mu_{D}}{\tilde{\mu}},
$$

where $K_{I}=\lambda_{I}+\frac{2}{3} \mu_{I}, K_{D}=\lambda_{D}+\frac{2}{3} \mu_{D}, \tilde{K}=\xi K_{I}+(1-\xi) K_{D}, \tilde{\mu}=\xi \mu_{I}+(1-\xi) \mu_{D}$. It is easy to see that for the intact material $(\xi=0)$ we have the mixture moduli equal to the ones of the intact material: $\lambda=\lambda_{I}, \mu=\mu_{I}$, while for the fully damaged material $(\xi=1)$ we obtain $\lambda=\lambda_{D}$, $\mu=\mu_{D}$.

The mixture-model averaging method of [103] applied to the definition of the rate of shear stress relaxation gives us the dependence of the shear stress relaxation time $\tau_{1}$ on the damage variable $\xi$ as follows:

$$
\tau_{1}=\left(\frac{1-\xi}{\tau_{I}}+\frac{\xi}{\tau_{D}}\right)^{-1}
$$

where $\tau_{I}$ and $\tau_{D}$ are the shear stress relaxation times for the intact and fully damaged materials respectively, which are usually highly nonlinear functions of the parameters of state. The particular choice of $\tau_{I}$ and $\tau_{D}$ that is used in this paper reads as

$$
\tau_{I}=\tau_{I 0} \exp \left(\alpha_{I}-\beta_{I}(1-\xi) Y\right), \quad \tau_{D}=\tau_{D 0} \exp \left(\alpha_{D}-\beta_{D} \xi Y\right),
$$

where $Y$ is the equivalent stress (e.g. the Von Mises stress), while $\tau_{I 0}, \alpha_{I}, \beta_{I}, \tau_{D 0}, \alpha_{D}, \beta_{D}$ are constants.

The parameter $\theta$ governing the rate of damage is also a nonlinear function of the parameters of state and in our numerical examples we take it in the following form:

$$
\theta=\theta_{0}(1-\xi)\left(\xi+\xi_{\epsilon}\right)\left[(1-\xi)\left(\frac{Y}{Y_{0}}\right)^{a}+\xi\left(\frac{Y}{Y_{1}}\right)\right]
$$

where $\xi_{\epsilon}, Y_{0}$ and $Y_{1}, a$ are constants. $\xi_{\epsilon}$ is usually taken as $10^{-16}$ in order to provide the growth of $\xi$ with the initial data $\xi=0$. 
The derivative $E_{\xi}$ can be computed with the use of $(3),(4),(8)$ and reads as

$$
\frac{\partial E}{\partial \xi}=-\frac{1}{2 \rho_{0}} \frac{\left(K_{I}-K_{D}\right) K_{I} K_{D}}{\tilde{K}^{2}}\left(1-\frac{\rho}{\rho_{0}}\right)^{2}-\frac{1}{4 \rho_{0}} \frac{\left(\mu_{I}-\mu_{D}\right) \mu_{I} \mu_{D}}{\tilde{\mu}^{2}} \stackrel{\circ}{G}_{i j} \stackrel{\circ}{G}_{i j}
$$

For the sake of simplicity we assume that the material parameters characterizing thermal properties of intact and damaged material are the same, that means that $c_{v}, c_{h}^{2}$ are constant and do not depend on $\xi$. The function $\theta_{2}$, characterizing the rate of heat flux relaxation, is taken as $\theta_{2}\left(\tau_{2}\right)=\tau_{2} \frac{c_{h}^{2}}{\rho T}$ that yields the classical Fourier heat conduction law with the thermal conductivity coefficient $\kappa=\tau_{2} c_{h}^{2}$ in the stiff relaxation limit $\left(\tau_{2} \rightarrow 0\right)$ [47].

\subsection{Discussion}

The model of Section 2.1 generalizes the unified model of continuum mechanics presented in $[96,47]$, taking into account also material damage processes and the possibility to simulate moving free surface problems via the use of a diffuse interface method (DIM) that simply employs a scalar colour function $\alpha$ in order to define where the solid is present $(\alpha=1)$ and where it is not $(\alpha=0)$, see [115]. The additional evolution equations for the material parameters (2) are added in order to capture correctly the motion of a continuum with heterogeneous material parameters undergoing large deformations. Note, that the governing equations for the heat transfer in the present model differ from that considered in [47] and are taken in the form originally proposed in [106]. The latter formulation of the heat transfer processes seems to be more natural, because it can be derived by the minimization of a Lagrangian and is in agreement with the Hamiltonian GENERIC formulation [97], as all equations from the general SHTC class.

As is noted in Section 2.1, if $\alpha=1$ then the PDE system (1) is a hyperbolic thermodynamically compatible system and advanced high-order methods can be applied to solve these equations. Nevertheless, there are very stiff algebraic source terms in the equations for distortion, thermal impulse and damage variable which create a significant difficulty for numerical computations. The exponential ODE integrator presented below helps to avoid problems related to the stiffness of the algebraic source terms in the governing PDE system and a series of numerical test problems has been solved successfully with the use of high order ADER-DG schemes with a posteriori subcell finite volume limiter method in conjunction with adaptive mesh refinement (AMR).

Another difficulty is an application of the model of damaged medium to the solution of real problems. It relates to the definition of the constitutive relations and, in particular, in the definition of the function $\theta$ characterizing the rate of change of the damage variable, as well as the shear and heat flux relaxation parameters $\theta_{1}$, and $\theta_{2}$. On the one hand, the material constants in the equation of state of the intact elastic medium can in principle be found from experimental measurements, which means that the Lamé constants $\lambda_{I}, \mu_{I}$ and the heat capacity $c_{v}$ can be considered as known. On the other hand, there is no way to get the sample of the fully damaged medium which appeared as a result of the deformation of the medium, and hence there seems to be no possibility to obtain the material constants of the damaged material by direct measurements.

The description of the damaged material requires not only material constants in the equation of state, but also a constitutive relations for the shear stress relaxation time $\tau_{1}$ and for the parameter $\theta$ governing the rate of the damage. All these characteristics of the medium can in principle be found with the use of experimental stress-strain diagrams, which are usually available from standard traction, torsion and compression experiments in solid mechanics. The method consists in doing a series of numerical computations and obtaining a set of stress-strain diagrams numerically, and then by variation of the material constants try to fit as much as possible the available experimental diagrams. Such a procedure was successfully used in the past for the closure of the nonlinear elastoplastic Godunov-Romenski model and the idea how to do this can be found in [67]. Recently 
this method has also been used for the closure of complex elastoplastic media with hardening, see [10].

The dynamic behavior of damaged materials is very complex and depends on the type of the medium. The damage followed by fracture can be brittle, or brittle-ductile, which means that the stress-strain diagrams can be completely different for different materials. In Section 3.5.2 two typical examples of brittle and ductile material behavior are presented. In our model, these diagrams can also depend on the strain rate, which is also shown in the same section. In this paper we do not calibrate the model parameters explicitly for such a dependence, since there is no experimental data available. Nevertheless, the constitutive relations chosen in Section 2.2 and the set of material constants presented in the Section on numerical tests gives good results in all considered test problems.

Last but not least, our thermodynamically compatible approach to material failure naturally includes also the phenomenon of material fatigue, i.e. the reduced resistance of the material to stress applied over a very large number of load cycles, see Section 3.5.2.

\section{Space-time adaptive ADER discontinuous Galerkin finite element schemes with a posteriori subcell finite volume limiter}

The equations (1b)-(1h) of the GPR model described above can be written in the following general form of a nonlinear system of hyperbolic PDEs with non-conservative products and stiff source terms:

$$
\frac{\partial \mathbf{Q}}{\partial t}+\nabla \cdot \mathbf{F}(\mathbf{Q})+\mathcal{B}(\mathbf{Q}) \cdot \nabla \mathbf{Q}=\mathbf{S}(\mathbf{Q}),
$$

where $\mathbf{Q}=\mathbf{Q}(\mathbf{x}, t)$ is the state vector; $\mathbf{x}=(x, y, z) \in \Omega \subset \mathbb{R}^{d}$ is the vector of spatial coordinates and $\Omega$ denotes the computational domain in $d$ space dimensions; $\mathbf{F}(\mathbf{Q})=(\mathbf{f}, \mathbf{g}, \mathbf{h})$ is the nonlinear flux tensor that contains the conservative part of the PDE system and $\mathcal{B}(\mathbf{Q}) \cdot \nabla \mathbf{Q}$ is a genuinely non-conservative term. When written in quasilinear form, the system (13) becomes

$$
\frac{\partial \mathbf{Q}}{\partial t}+\mathcal{A}(\mathbf{Q}) \cdot \nabla \mathbf{Q}=\mathbf{S}(\mathbf{Q}),
$$

where the matrix $\mathcal{A}(\mathbf{Q})=\partial \mathbf{F}(\mathbf{Q}) / \partial \mathbf{Q}+\mathcal{B}(\mathbf{Q})$ includes both the Jacobian of the conservative flux, as well as the non-conservative product. The hyperbolicity of system (14) has been discussed in [96]. However, for the practical implementation of the numerical schemes used in this paper, the eigenvectors $\mathbf{R}_{n}$ of the matrix $\mathcal{A}_{n}=\mathcal{A}(\mathbf{Q}) \cdot \mathbf{n}$ ( $\mathbf{n}$ is a unit-normal vector) will not be needed, even if they were in principle available.

The PDE system (13) is solved by resorting to a high order one-step ADER-FV and ADER-DG method $[100,39,41]$, which provides at the same time high order of accuracy in both space and time in one single step, hence completely avoiding the Runge-Kutta sub-stages that are typically used in Runge-Kutta DG and Runge-Kutta WENO schemes. The method will be presented in the unified framework of $P_{N} P_{M}$ methods introduced in [39], which contains both, DG schemes and FV schemes as special cases of a more general class of methods. For related work on $P_{N} P_{M}$ schemes, the reader is referred to $[82,83]$. The construction of fully-discrete high order one-step schemes is typical of the ADER approach $[120,119,124]$. In the following, we only summarize the main steps, while for more details the reader is referred to [39, 50, 71, 59, 9, 52, 132, 131, 24].

\subsection{Data representation and reconstruction}

The computational domain $\Omega$ is discretized by a computational mesh that can be structured or unstructured, composed of conforming elements denoted by $T_{i}$, where the index $i$ ranges from 1 to the total number of elements $N_{E}$. We will further denote the volume (area) of an individual 
cell by $\left|T_{i}\right|=\int_{T_{i}} d \mathbf{x}$. The discrete solution of PDE (13) is denoted by $\mathbf{u}_{h}\left(\mathbf{x}, t^{n}\right)$ and is represented by piecewise polynomials of maximum degree $N \geq 0$. Within each cell $T_{i}$ we have

$$
\mathbf{u}_{h}\left(\mathbf{x}, t^{n}\right)=\sum_{l}^{\mathcal{N}} \Phi_{l}(\mathbf{x}) \hat{\mathbf{u}}_{l, i}^{n}:=\Phi_{l}(\mathbf{x}) \hat{\mathbf{u}}_{l, i}^{n}, \quad \mathbf{x} \in T_{i},
$$

where we have introduced the classical Einstein summation convention over two repeated indices. The discrete solution $\mathbf{u}_{h}\left(\mathbf{x}, t^{n}\right)$ is defined in the space of piecewise polynomials up to degree $N$, spanned by a set of basis functions $\Phi_{l}=\Phi_{l}(\mathbf{x})$. Throughout this paper we use a tensor-product nodal basis for quadrilateral and hexahedral elements. The nodal basis is given by the Lagrange interpolation polynomials passing through the Gauss-Legendre quadrature nodes on the unit element $[0,1]^{d}$, see [113] for details on multidimensional quadrature. The symbol $\mathcal{N}$ denotes the number of degrees of freedom per element and is given by $\mathcal{N}=(N+1)^{d}$ for quadrilateral / hexahedral Cartesian elements in $d$ space dimensions. In the framework of $P_{N} P_{M}$ methods, the discrete solution $\mathbf{u}_{h}$ is now reconstructed in order to obtain for each element a piecewise polynomial $\mathbf{w}_{h}(\mathbf{x}, t)$ of degree $M \geq N$, with a total number of $\mathcal{M}$ degrees of freedom. Details on the nonlinear WENO reconstruction and on the $P_{N} P_{M}$ reconstruction can be found in [44, 45, 39] and are not repeated here. The number of degrees of freedom $\mathcal{M}$ is again $\mathcal{M}=(M+1)^{d}$ for tensor-product elements in $d$ space dimensions, respectively. The reconstruction step is simply abbreviated by $\mathbf{w}_{h}(\mathbf{x}, t)=\mathcal{R}\left(\mathbf{u}_{h}(\mathbf{x}, t)\right)$, and the reconstruction polynomial $\mathbf{w}_{h}(\mathbf{x}, t)$ is written as

$$
\mathbf{w}_{h}\left(\mathbf{x}, t^{n}\right)=\sum_{l}^{\mathcal{M}} \Psi_{l}(\mathbf{x}) \hat{\mathbf{w}}_{l, i}^{n}:=\Psi_{l}(\mathbf{x}) \hat{\mathbf{w}}_{l, i}^{n}, \quad \mathbf{x} \in T_{i} .
$$

Note that for $N=M$ the $P_{N} P_{M}$ method reduces to a classical discontinuous Galerkin finite element scheme, with the reconstruction operator equal to the identity operator, $\mathcal{R}=\mathcal{I}$, or, equivalently, $\mathbf{w}_{h}\left(\mathbf{x}, t^{n}\right)=\mathbf{u}_{h}\left(\mathbf{x}, t^{n}\right)$, while for the case $N=0$ the method reduces to a standard high order WENO finite volume scheme, if a WENO reconstruction operator is adopted.

For WENO schemes on structured meshes we have found that it is particularly convenient to adopt one-dimensional stencils, each composed by $n_{e}=M+1$ cells, which are subsequently oriented along each spatial direction. The resulting reconstruction is still multidimensional, but implemented with a dimension-by-dimension strategy. A complete description of this approach can be found in $[51,129,24]$, including also the necessary details for the employed adaptive mesh refinement.

In this paper, however, we will only use the two special limits of the general $P_{N} P_{M}$ approach, i.e. either $N=0$ (pure FV) or $N=M$ (pure $\mathrm{DG}$ ), where the finite volume scheme is used under the form of an a posteriori subcell finite volume limiter, see [52, 132].

\subsection{Local space-time predictor}

The discrete solution $\mathbf{w}_{h}\left(\mathbf{x}, t^{n}\right)$ is now evolved in time according to an element-local weak formulation of the governing PDE in space-time, see [42, 39, 71, 50, 59, 9, 52, 132, 131, 24]. The local space-time Galerkin method is only used for the construction of an element-local predictor solution of the PDE in the small, hence neglecting the influence of neighbor elements. This predictor will subsequently be inserted into the corrector step described in the next section, which then provides the appropriate coupling between neighbor elements via a numerical flux function (Riemann solver) and a path-conservative jump term for the discretization of the non-conservative product. To simplify notation, we define

$$
\langle f, g\rangle=\int_{t^{n}}^{t^{n+1}} \int_{T_{i}} f(\mathbf{x}, t) g(\mathbf{x}, t) d \mathbf{x} d t, \quad[f, g]^{t}=\int_{T_{i}} f(\mathbf{x}, t) g(\mathbf{x}, t) d \mathbf{x}
$$


which denote the scalar products of two functions $f$ and $g$ over the space-time element $T_{i} \times\left[t^{n} ; t^{n+1}\right]$ and over the spatial element $T_{i}$ at time $t$, respectively. Within the local space-time predictor, the discrete solution of equation (13) is denoted by $\mathbf{q}_{h}=\mathbf{q}_{h}(\mathbf{x}, t)$. We then multiply (13) with a spacetime test function $\theta_{k}=\theta_{k}(\mathbf{x}, t)$ and subsequently integrate over the space-time control volume $T_{i} \times\left[t^{n} ; t^{n+1}\right]$. Inserting $\mathbf{q}_{h}$, the following weak formulation of the PDE is obtained:

$$
\left\langle\theta_{k}, \frac{\partial \mathbf{q}_{h}}{\partial t}\right\rangle+\left\langle\theta_{k}, \nabla \cdot \mathbf{F}\left(\mathbf{q}_{h}\right)+\mathcal{B}\left(\mathbf{q}_{h}\right) \cdot \nabla \mathbf{q}_{h}\right\rangle=\left\langle\theta_{k}, \mathbf{S}\left(\mathbf{q}_{h}\right)\right\rangle .
$$

The discrete representation of $\mathbf{q}_{h}$ in element $T_{i} \times\left[t^{n}, t^{n+1}\right]$ is assumed to have the following form

$$
\mathbf{q}_{h}=\mathbf{q}_{h}(\mathbf{x}, t)=\sum_{l} \theta_{l}(\mathbf{x}, t) \hat{\mathbf{q}}_{l, i}^{n}:=\theta_{l} \hat{\mathbf{q}}_{l, i}^{n}
$$

where $\theta_{l}(\mathbf{x}, t)$ is a space-time basis function of maximum degree $M$. For the basis functions $\theta_{l}$ we use a tensor-product of $1 \mathrm{D}$ nodal basis functions given by the Lagrange interpolation polynomials of the Gauss-Legendre quadrature points for Cartesian tensor-product elements. After integration by parts in time of the first term, Eqn. (18) reads

$$
\left[\theta_{k}, \mathbf{q}_{h}\right]^{t^{n+1}}-\left[\theta_{k}, \mathbf{w}_{h}\left(\mathbf{x}, t^{n}\right)\right]^{t^{n}}-\left\langle\frac{\partial}{\partial t} \theta_{k}, \mathbf{q}_{h}\right\rangle+\left\langle\theta_{k}, \nabla \cdot \mathbf{F}\left(\mathbf{q}_{h}\right)+\mathcal{B}\left(\mathbf{q}_{h}\right) \cdot \nabla \mathbf{q}_{h}\right\rangle=\left\langle\theta_{k}, \mathbf{S}\left(\mathbf{q}_{h}\right)\right\rangle .
$$

Note that the high order polynomial reconstruction of the $P_{N} P_{M}$ scheme $\mathbf{w}_{h}\left(\mathbf{x}, t^{n}\right)$ is taken into account in (20) in a weak sense by the term $\left[\theta_{k}, \mathbf{w}_{h}\left(\mathbf{x}, t^{n}\right)\right]^{t^{n}}$. This corresponds to the choice of a numerical flux in time direction, which is nothing else than upwinding in time, according to the causality principle.

Note further that due to the DG approximation in space-time, we may have $\mathbf{q}_{h}\left(\mathbf{x}, t^{n}\right) \neq$ $\mathbf{w}_{h}\left(\mathbf{x}, t^{n}\right)$ in general, hence the choice of a numerical flux in time direction is necessary. Note further that in (20) we have not used integration by parts in space, nor any other coupling to spatial neighbor elements. The integrals appearing in the weak form (20), as well as the space-time test and basis functions involved are conveniently written by making use of a space-time reference element $T_{e} \times[0 ; 1]$.

The solution of (20) yields the unknown space-time degrees of freedom $\hat{\mathbf{q}}_{l, i}^{n}$ for each space-time element $T_{i} \times\left[t^{n} ; t^{n+1}\right]$ and is easily achieved with a fast converging iterative scheme, see [39, 71, 50] for more details. In [75] it was proven that the resulting iteration matrix is nilpotent, which explains the convergence to the exact solution in a finite number of iterations for linear homogeneous systems already observed in [39]. In [24] a more general convergence proof of the space-time predictor based on fixed point arguments was given for nonlinear conservation laws. The above space-time Galerkin predictor has replaced the cumbersome Cauchy-Kovalewski procedure that has been initially employed in the original version of ADER finite volume and ADER discontinuous Galerkin schemes [109, 119, 124, 120, 46, 114, 45]. Note that very recently, a new reformulation of the ADER method has been proposed, where the reconstruction and time evolution steps are performed in terms of the vector of primitive variables $\mathbf{V}$ instead of using the vector $\mathbf{Q}$ of conserved quantities, see [130] and also [99], for a similar approach in the context of Runge-Kutta WENO FV schemes.

\subsection{Fully discrete one-step finite volume and discontinuous Galerkin schemes}

At the aid of the local space-time predictor $\mathbf{q}_{h}$, a fully discrete one-step $P_{N} P_{M}$ scheme can now be simply obtained by multiplication of the governing PDE system (13) by test functions $\Phi_{k}$ from the space of piecewise polynomials up to degree $N$, which are identical with the spatial basis functions of the original data representation before reconstruction, and subsequent integration over 
the space-time control volume $T_{i} \times\left[t^{n} ; t^{n+1}\right]$. Due to the presence of non-conservative products, the jumps of $\mathbf{q}_{h}$ across element boundaries are taken into account in the framework of path-conservative schemes put forward by Castro and Parés in the finite volume context [27, 94] and subsequently extended to DG schemes in [104] and [41, 43], where also a generalization to the unified $P_{N} P_{M}$ framework has been provided. All these approaches are based on the theory of Dal Maso, Le Floch and Murat [85], which gives a definition of weak solutions in the context of non-conservative hyperbolic PDE. For open problems concerning path-conservative schemes, the reader is referred to $[3,28]$.

If $\mathbf{n}$ is the outward pointing unit normal vector on the surface $\partial T_{i}$ of element $T_{i}$ and the pathconservative jump term in normal direction is denoted by $\mathcal{D}_{h}^{-}\left(\mathbf{q}_{h}^{-}, \mathbf{q}_{h}^{+}\right) \cdot \mathbf{n}$, which is a function of the left and right boundary-extrapolated data, $\mathbf{q}_{h}^{-}$and $\mathbf{q}_{h}^{+}$, respectively, then we obtain the following path-conservative one-step $P_{N} P_{M}$ scheme, see [41]:

$$
\begin{array}{r}
\left(\int_{T_{i}} \Phi_{k} \Phi_{l} d \mathbf{x}\right)\left(\hat{\mathbf{u}}_{l}^{n+1}-\hat{\mathbf{u}}_{l}^{n}\right)+\int_{t^{n}} \int_{\partial T_{i}} \Phi_{k} \mathcal{D}_{h}^{-}\left(\mathbf{q}_{h}^{-}, \mathbf{q}_{h}^{+}\right) \cdot \mathbf{n} d S d t \\
+\int_{t^{n}}^{t^{n+1}} \int_{T_{i} \backslash \partial T_{i}} \Phi_{k}\left(\nabla \cdot \mathbf{F}\left(\mathbf{q}_{h}\right)+\mathcal{B}\left(\mathbf{q}_{h}\right) \cdot \nabla \mathbf{q}_{h}\right) d \mathbf{x} d t=\int_{t^{n}}^{t^{n+1}} \int_{T_{i}} \Phi_{k} \mathbf{S}\left(\mathbf{q}_{h}\right) d \mathbf{x} d t .
\end{array}
$$

The element mass matrix appears in the first integral of (21), the second term accounts for the jump in the discrete solution at element boundaries and the third term takes into account the smooth part of the non-conservative product. For general complex nonlinear hyperbolic PDE systems we use the simple Rusanov method [108] (also called the local Lax Friedrichs method), although any other kind of Riemann solver could be also used, see [122] for an overview of stateof-the-art Riemann solvers. In these regards, we would also like to point out the new general reformulation of the HLLEM Riemann solver of Einfeldt and Munz [53, 54], within the setting of path-conservative schemes recently forwarded in [40], as well as the family of MUSTA schemes, which has been applied to the equations of nonlinear elasticity in [118].

The extension of the Rusanov flux to the path-conservative framework requires the introduction of an additional nonconservative jump term and reads

$$
\mathcal{D}_{h}^{-}\left(\mathbf{q}_{h}^{-}, \mathbf{q}_{h}^{+}\right) \cdot \mathbf{n}=\frac{1}{2}\left(\mathbf{F}\left(\mathbf{q}_{h}^{+}\right)-\mathbf{F}\left(\mathbf{q}_{h}^{-}\right)\right) \cdot \mathbf{n}+\frac{1}{2}\left(\tilde{\mathcal{B}} \cdot \mathbf{n}-s_{\max } \mathbf{I}\right)\left(\mathbf{q}_{h}^{+}-\mathbf{q}_{h}^{-}\right),
$$

with the maximum signal speed at the element interface $s_{\max }=\max \left(\left|\boldsymbol{\Lambda}\left(\mathbf{q}_{h}^{+}\right)\right|,\left|\boldsymbol{\Lambda}\left(\mathbf{q}_{h}^{-}\right)\right|\right)$and the matrix $\tilde{\mathcal{B}} \cdot \mathbf{n}$ given by the following path-integral along a straight line segment path $\psi$ :

$$
\tilde{\mathcal{B}} \cdot \mathbf{n}=\int_{0}^{1} \mathcal{B}\left(\psi\left(\mathbf{q}_{h}^{-}, \mathbf{q}_{h}^{+}, s\right) \cdot \mathbf{n} d s, \quad \psi\left(\mathbf{q}_{h}^{-}, \mathbf{q}_{h}^{+}, s\right)=\mathbf{q}_{h}^{-}+s\left(\mathbf{q}_{h}^{+}-\mathbf{q}_{h}^{-}\right) .\right.
$$

According to the suggestions made in $[41,43,48,26,49]$, the path-integrals can be conveniently evaluated numerically by the use of a classical Gauss-Legendre quadrature formula on the unit interval $[0 ; 1]$. For an alternative choice of the path, see $[88,89]$.

This completes the brief description of the $P_{N} P_{M}$ scheme used for the discretization of the governing PDE system (13).

In the case of ADER finite volume schemes, we simply have $N=0, \mathcal{N}=1, \Phi_{k}=1$, and the limiter is directly incorporated in the nonlinear reconstruction operator $\mathbf{w}_{h}\left(\mathbf{x}, t^{n}\right)=\mathcal{R}\left(\mathbf{u}_{h}\left(\mathbf{x}, t^{n}\right)\right)$. 
For ADER discontinuous Galerkin finite element schemes $\left(N=M, \Phi_{k}=\Psi_{k}\right)$ a new family of a posteriori sub-cell finite volume limiters has been forwarded in [52, 132, 131] and is employed throughout this work. In particular, in this paper the subcell finite volume limiter employs simple piecewise linear reconstruction based on the minmod slope limiter. For alternative finite volume subcell limiters in the context of DG schemes, see the work of Sonntag \& Munz [110] and Meister \& Ortleb [86]. For details on the adaptive mesh refinement strategy used in this paper, the reader is referred to $[51,132,131,23,126,101]$.

\subsection{Treatment of stiff source terms via exponential time integration}

Due to the strong nonlinearities present in the reaction-like kinetics characterising the evolution of the material damage variable $\xi$, a special treatment of the stiff source term is required. Note that the exponential time integration method described in this section is applied only within the subcell finite volume limiter scheme.

Specifically, the rupture process develops at time scales that are much quicker than what given by the CFL condition for elastic waves in the intact medium and thus the effects of the stiff source terms might compromise the stability of the simulations.

In order to solve the issue, we make use of a split treatment of the source, that is, at each timestep we solve the governing equations neglecting the source terms for strain relaxation and rupture dynamics, obtaining a preliminary solution, to be used in a second step as initial condition for a Cauchy problem constructed by neglecting all the differential terms appearing in the PDE system, except for time derivatives. Then one can evolve in time the pointwise preliminary solution by solving such initial value problem between the current time level and the following one.

Unfortunately, the integration of the ordinary differential equations arising from the split treatment of the algebraic source term cannot be tackled by under-resolving the sub-timestep evolution of the damage variable $\xi$ and the distortion matrix $\mathbf{A}$ with a simple implicit Euler scheme. Instead we decided to employ an efficient exponential integration technique that is not only more robust, but also more accurate.

In the following we present the procedure for applying the exponential integrator to a general first order system of ordinary differential equations like

$$
\frac{\mathrm{d} \mathbf{Q}}{\mathrm{d} t}=\mathbf{S}(\mathbf{Q}, t)
$$

for which we write a linearisation about a given state $\mathbf{Q}^{*}$ and time $t^{*}$ as

$$
\frac{\mathrm{d} \mathbf{Q}}{\mathrm{d} t}=\mathbf{B}^{*}+\mathbf{J}^{*}\left(\mathbf{Q}^{*}, t^{*}\right)\left(\mathbf{Q}-\mathbf{Q}^{*}\right)
$$

In this work, the vector $\mathbf{Q}$ will have ten components, the first being the material damage variable $\xi$, followed by the nine entries of the distortion matrix $\mathbf{A}$. One then defines the Jacobian matrix of the source $\mathbf{J}^{*}=\mathbf{J}\left(\mathbf{Q}^{*}, t^{*}\right)=\partial \mathbf{S} / \partial \mathbf{Q}$ and in the same way the source vector evaluated at the linearisation state is $\mathbf{B}^{*}=\mathbf{S}\left(\mathbf{Q}^{*}, t^{*}\right)$. Furthermore, we introduce the vector

$$
\mathbf{C}^{*}=\mathbf{C}^{*}\left(\mathbf{B}^{*}, \mathbf{J}^{*}\right)=\mathbf{C}^{*}\left(\mathbf{Q}^{*}, t^{*}\right)
$$

which will be used as an indicator for the adaptive timestepping scheme. This vector may be composed for example by the the entries of the matrix $\mathbf{J}^{*}$, together with all the elements of the vector $\mathbf{B}^{*}$ and the state $\mathbf{Q}^{*}$. A simpler general choice might be using only the state vector, but we recommend that some relevant combination of the listed variables be included, to indicate changes in the nature or in the magnitude of the linearised source operator. For the tests presented in this work the vector of indicator variables is comprised simply of the linearisation state $\mathbf{Q}^{*}$ and of two characteristic relaxation times for the damage variable equation and for the strain relaxation 
equation. It is then necessary to compute an exact analytical solution of the linear nonhomogeneous Cauchy problem

$$
\left\{\begin{array}{l}
\frac{\mathrm{d} \mathbf{Q}}{\mathrm{d} t}=\mathbf{S}^{*}\left(\mathbf{Q} ; \mathbf{Q}^{*}, t^{*}\right)=\mathbf{B}^{*}+\mathbf{J}^{*}\left(\mathbf{Q}^{*}, t^{*}\right)\left(\mathbf{Q}-\mathbf{Q}^{*}\right), \\
\mathbf{Q}\left(t_{n}\right)=\mathbf{Q}_{n},
\end{array}\right.
$$

and in the general case, one can use the algorithms of Higham [72] and Al-Mohy and Higham [4, 5] for a robust evaluation of matrix exponentials needed for such computations. More notes on the solution of (27) will be given in Section 3.5.1.

We will set $\mathbf{Q}_{\mathrm{e}}\left(t ; \mathbf{S}^{*}, t_{n}, \mathbf{Q}_{n}\right)$ to denote the analytical solution of $(27)$, and, as for $\mathbf{S}^{*}\left(\mathbf{Q} ; \mathbf{Q}^{*}, t^{*}\right)$, the semicolon will separate the variable on which $\mathbf{Q}_{\mathbf{e}}$ and $\mathbf{S}^{*}$ depend continuously ( $t$ or $\mathbf{Q}$ ) from the fixed parameters used to build the operators. The state vector at timestep $t_{n}$ is $\mathbf{Q}_{n}$, and the variable timestep size is $\Delta t^{n}=t_{n+1}-t_{n}$.

\subsubsection{Timestepping}

Marching from a start time $t_{0}$ to an end time $t^{\text {end }}$ is carried out as follows. First, an initial timestep size $\Delta t^{0}$ is chosen; then, at each timestep, the state $\mathbf{Q}_{n+1}$ at the new time level $t_{n+1}$ can be computed by means of an iterative procedure which will terminate by yielding a value for $\mathbf{Q}_{n+1}$, as well as a new timestep size $\Delta t^{n+1}=t_{n+2}-t_{n+1}$ based on an estimator embedded in the iterative solution algorithm. There is also the possibility that, due to the timestep size $\Delta t$ being too large, the value of $\mathbf{Q}_{n+1}$ be flagged as not acceptable. In this case the procedure returns a reduced timestep size for the current timestep $\Delta t^{n}=t_{n+1}-t_{n}$ and the solution for $\mathbf{Q}_{n+1}$ will be attempted again using this reduced timestep size. Specifically, in practice we set the new value of $\Delta t^{n}$ to be half of the one used in the previous attempt.

\subsection{Iterative computation of the solution during one timestep}

At each iteration of index $k$ an average state vector is computed as

$$
\mathbf{Q}_{n+1 / 2}^{* k}=\frac{1}{2}\left(\mathbf{Q}_{n}+\mathbf{Q}_{n+1}^{* k-1}\right)
$$

and this midpoint state is formally associated with an intermediate time level

$$
t_{n+1 / 2}=\frac{1}{2}\left(t_{n}+t_{n+1}\right) .
$$

For the first iteration we provide a guess value for $\mathbf{Q}_{n+1}^{* k-1}$, with the simplest choice being given by $\mathbf{Q}_{n+1}^{* k-1}=\mathbf{Q}_{n}$. Then the coefficients $\mathbf{C}_{n+1 / 2}^{* k}$ are computed as

$$
\mathbf{C}_{n+1 / 2}^{* k}=\mathbf{C}_{n+1 / 2}^{* k}\left(\mathbf{Q}_{n+1 / 2}^{* k}, t_{n+1 / 2}\right),
$$

and concurrently (that is, within the same code block), one can build the affine source operator

$$
\mathbf{S}_{n+1 / 2}^{* k}=\mathbf{S}_{n+1 / 2}^{* k}\left(\mathbf{Q} ; \mathbf{Q}_{n+1 / 2}^{* k}, t_{n+1 / 2}\right),
$$

then one has to find the solution of the initial value problem for the linearised equations

$$
\left\{\begin{array}{l}
\frac{\mathrm{d} \mathbf{Q}}{\mathrm{d} t}=\mathbf{S}_{n+1 / 2}^{* k}\left(\mathbf{Q} ; \mathbf{Q}_{n+1 / 2}^{* k}, t_{n+1 / 2}\right), \\
\mathbf{Q}\left(t_{n}\right)=\mathbf{Q}_{n}
\end{array}\right.
$$


yielding the updated state vector for the next iteration

$$
\mathbf{Q}_{n+1}^{* k}=\mathbf{Q}_{\mathrm{e}}\left(t_{n+1} ; \mathbf{S}_{n+1 / 2}^{* k}, t_{n}, \mathbf{Q}_{n}\right) \text {. }
$$

It is then checked that the state vector $\mathbf{Q}_{n+1}^{* k}$ be physically admissible: in our case this means verifying that the damage variable is in the unit interval, i.e. $\xi \in[0,1]$. Also, one can check that floating-point exceptions be absent. Additionally, one must evaluate

$$
\mathbf{C}_{n+1}^{* k}=\mathbf{C}_{n+1}^{* k}\left(\mathbf{Q}_{n+1}^{* k}, t_{n+1}\right),
$$

this vector of coefficients will not be employed for the construction of an affine source operator $\mathbf{S}_{n+1}^{* k}$, but only for checking the validity of the solution obtained from (32), by comparing the coefficients vector $\mathbf{C}_{n+1}^{* k}$ to $\mathbf{C}_{n}^{*}$, as well as comparing the coefficients $\mathbf{C}_{n+1 / 2}^{* k}$ used in the middlepoint affine operator for the initial coefficients $\mathbf{C}_{n}{ }_{n}$. At the end of the iterative procedure, one will set $\mathbf{C}^{*}{ }_{n+1}=\mathbf{C}_{n+1}^{* k}$, so that this can be reused as the new reference vector of coefficients for the next timestep.

The convergence criterion for stopping the iterative computation is implemented by evaluating

$$
r=\max \left(\frac{\left|\mathbf{Q}_{n+1}^{* k}-\mathbf{Q}_{n+1}^{* k-1}\right|}{\left|\mathbf{Q}_{n+1}^{* k}\right|+\left|\mathbf{Q}_{n+1}^{* k-1}\right|+\epsilon_{r}}\right),
$$

and checking whether $r \leq r_{\max }$, with $r_{\max }$ and $\epsilon_{r}$ given tolerances, or alternatively when the iteration count $k$ has reached a fixed maximum value $k_{\max }$. Moreover, we found convenient to limit the maximum number of iterations allowed, and specifically we set $k_{\max }=8$, or alternatively one can decide to use $k_{\max }=3$ and flag the state vector $\mathbf{Q}_{n+1}^{* k}$ as not admissible, as if a floatingpoint exception had been triggered, whenever the iterative procedure terminates by reaching the maximum iteration count. When convergence is finally obtained, in order to test if the Cauchy problem

$$
\left\{\begin{array}{l}
\frac{\mathrm{d} \mathbf{Q}}{\mathrm{d} t}=\mathbf{S}(\mathbf{Q}, t), \\
\mathbf{Q}\left(t_{n}\right)=\mathbf{Q}_{n} .
\end{array}\right.
$$

is well approximated by its linearised version (32), we compute two error metrics

$$
\begin{aligned}
& \delta_{n+1 / 2}=\max \left(\frac{\left|\mathbf{C}^{*}{ }_{n+1 / 2}-\mathbf{C}^{*}{ }_{n}\right|}{\left|\mathbf{C}^{*}{ }_{n+1 / 2}\right|+\left|\mathbf{C}^{*}{ }_{n}\right|+\epsilon_{\delta}}\right), \\
& \delta_{n+1}=\max \left(\frac{\left|\mathbf{C}^{*}{ }_{n+1}-\mathbf{C}^{*}{ }_{n}\right|}{\left|\mathbf{C}^{*}{ }_{n+1}\right|+\left|\mathbf{C}^{*}{ }_{n}\right|+\epsilon_{\delta}}\right),
\end{aligned}
$$

and we verify if

$$
\delta=\max \left(\delta_{n+1 / 2}, \delta_{n+1}\right) \leq \delta_{\max } .
$$

Note that one should specify a tolerance $\delta_{\max }$ for (39) and a floor value $\epsilon_{\delta}$, which is used in order to prevent that excessive precision requirements be imposed in those situations when all the coefficients are so small than even large relative variations expressed by Equations (37) and (38) do not affect the solution in a significant manner. If $\delta \leq \delta_{\max }$, then the state vector at the new time level is set to be $\mathbf{Q}_{n+1}=\mathbf{Q}_{n+1}^{* k}$ and a new timestep size can be computed as

$$
\Delta t_{n+1}=\lambda \frac{\delta_{\max }}{\delta+\epsilon}, \quad \text { with } \quad \lambda=0.8, \quad \epsilon=10^{-14},
$$


otherwise a new attempt at the solution of (36) is carried out, with a halved timestep size. The same will happens in case at any time the admissibility test on $\mathbf{Q}_{n+1}^{* k}$ fails.

The resulting ODE integrator is second order accurate, that is, the error in the solution decreases quadratically with the number of timesteps employed, and, thanks to the piecewiseexponential ansatz, yields by construction very good results compared to a standard second order pieciewise linear evolution of the state vector, especially in the integration steps in which the equations are only weakly nonlinear, i.e. away from rupture faults and cracks.

\subsubsection{Solution of the linearised problem}

If the system of linearised equations is large, it might be infeasible to explicitly write a closed form expression for the solution of the linearised initial value problem (27), which can be written compactly as

$$
\mathbf{Q}(t)=\exp \left(\mathbf{J}^{*}\left(t-t_{n}\right)\right)\left(\mathbf{Q}\left(t_{n}\right)+\left(\mathbf{J}^{*}\right)^{-1} \mathbf{B}^{*}-\mathbf{Q}^{*}\right)-\left(\mathbf{J}^{*}\right)^{-1} \mathbf{B}^{*}+\mathbf{Q}^{*},
$$

but the evaluation of the matrix exponential and the computation of the inverse Jacobian $\left(\mathbf{J}^{*}\right)^{-1}$ in general need to be carried out numerically, and in particular the inversion of $\mathbf{J}^{*}$ may constitute an ill-conditioned problem. Specifically, the timescale associated with strain relaxation can be significantly different from the reaction speed of material failure. This issue can be treated by detecting those rows of the Jacobian that have much smaller entries than a given global scale for the full system and removing them from the matrix, then inverting a reduced system of equations with better conditioning.

An alternative approach, used in this work, consists in constructing an approximate linearisation of the source Jacobian so that it is structured in two independent blocks. One is a scalar equation for $\xi$ obtained by suppressing all the off-diagonal entries of $\mathbf{J}^{*}$. The other, analogously, can be obtained from removing all dependencies on the damage variable of the strain-relaxation subsystem. This way, one can compute the analytical solution for the two subsystems separately, and since the two blocks are built so that they are independent on each other, these will constitute an approximate
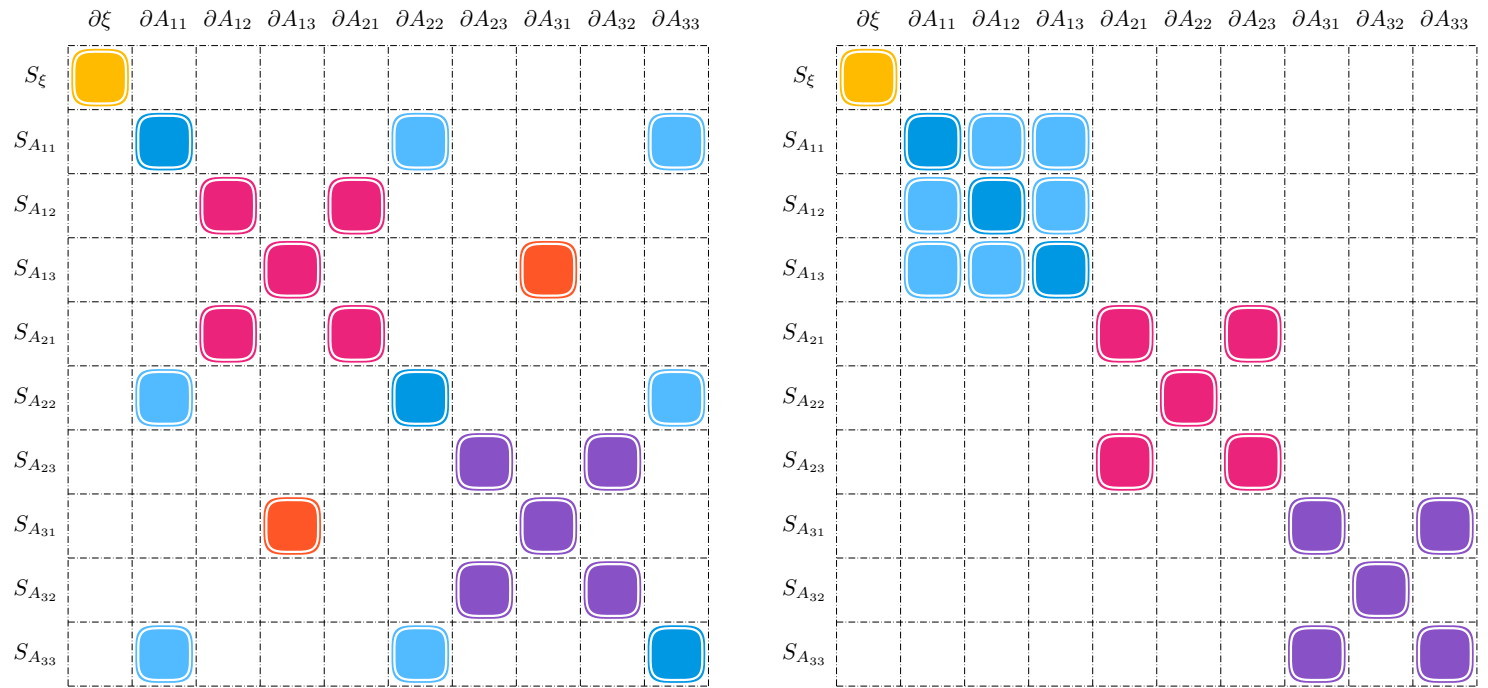

Figure 1: Structure of the two-block split (material failure and strain relaxation) of the source Jacobian (on the left) and of the four-block split (material failure and three small submatrices for strain relaxation) of the system (on the right). 
solution of (27). Moreover, being the fracture kinetics subsystem composed of a single scalar equation, the evaluation of the analytical solution can use the standard scalar exponential and division operations, instead of the more delicate procedures for computing matrix exponentials and especially inverting matrices. As an added benefit, if one approximates the relaxation timescale $\tau_{m}$ with a constant value (to be recomputed iteratively), the Jacobian for the strain relaxation subsystem can be evaluated analytically in a very efficient manner, without resorting to numerical differentiation.

In some particular cases, another approximation step can be taken: whenever the off diagonal entries of the distortion matrix are small in comparison to the diagonal ones, many of the entries of the the strain-relaxation source Jacobian become negligible and one can exploit the sparsity of the strain-relaxation Jacobian matrix to further split it into three blocks. In detail, we take the material-damage/strain-relaxation source vector

$$
\mathbf{S}(\mathbf{Q})=\left(S_{\xi}, S_{A_{11}}, S_{A_{12}}, S_{A_{13}}, S_{A_{21}}, S_{A_{22}}, S_{A_{23}}, S_{A_{31}}, S_{A_{32}}, S_{A_{33}}\right)^{\top}
$$

and rearrange it as four independent sources

$$
\begin{aligned}
& \mathbf{S}_{\mathrm{a}}=\left(S_{\xi}\right)^{\top}, \\
& \mathbf{S}_{\mathrm{b}}=\left(\begin{array}{lll}
S_{A_{11}}, & S_{A_{22}}, & S_{A_{33}}
\end{array}\right)^{\top}, \\
& \mathbf{S}_{\mathrm{c}}=\left(\begin{array}{lll}
S_{A_{12}}, & S_{A_{13}}, & \left.S_{A_{21}}\right)^{\top}, \\
\mathbf{S}_{\mathrm{d}}=\left(S_{A_{23}},\right. & S_{A_{31}}, & S_{A_{32}}
\end{array}\right)^{\top} .
\end{aligned}
$$

Then of each one of the sources we compute the Jacobian with respect to only the variables in the corresponding block, that is, for example $\mathbf{S}_{\mathrm{b}}$ will be differentiated only with respect to the diagonal components of $\mathbf{A}$ and all off-diagonal-block contributions to the global Jacobian like $\partial S_{A_{11}} / \partial A_{12}$ will be assumed null. This approximation is justified (under the assumption that offdiagonal components of $\mathbf{A}$ be small) for all off-diagonal-block derivatives except for $\partial S_{A_{13}} / \partial A_{31}$ and $\partial S_{A_{31}} / \partial A_{13}$, which maintain a large magnitude even when $\mathbf{A}$ is almost diagonal. These elements can be suppressed regardless, as we already do for all the derivatives like $\partial S_{\xi} / \partial A_{i j}$ and $\partial S_{A_{i j}} / \partial \xi$, relying on the adaptive timestepping method and on the iterative relinearisation for the task of reintroducing the lost coupling terms. A visual representation of the two-block split (material failure and strain relaxation) and the four-block split (material failure and three small submatrices for strain relaxation) of the source Jacobian is given in Figure 1.

\subsubsection{Examples, validation against LSODA and comparison with the implicit Euler scheme}

Here we validate our exponential time integrator against available standard software for the numerical integration of stiff ODE. In particular, we compare against the community standard LSODA/ODEPACK, see $[73,74]$. We also compare our exponential time integrator against a simple implicit Euler time integration scheme, showing clearly that implicit Euler time stepping, even with very fine substeps, can lead to significantly inaccurate results.

The benchmark consists in computing the stress-strain diagram for a given choice of spatially homogeneous material parameters: we distort, with constant strain rate $\dot{\boldsymbol{\epsilon}}$, a homogeneous sample of material, which is initially unstressed $(\mathbf{A}=\mathbf{I})$ and undamaged $(\xi=0)$. The governing PDE system (1d) therefore reduces to a simple ODE system. The strain rate tensor will have the form

$$
\dot{\boldsymbol{\epsilon}}(t)=\left(\begin{array}{ccc}
\dot{\epsilon}_{11}(t) & 0 & 0 \\
0 & 0 & 0 \\
0 & 0 & 0
\end{array}\right),
$$


and the resulting governing ODE system for the dynamics reads

$$
\begin{aligned}
& \frac{\mathrm{d} \xi}{\mathrm{d} t}=-\theta \frac{\partial E}{\partial \xi}, \\
& \frac{\mathrm{d} \mathbf{A}}{\mathrm{d} t}=-\mathbf{A} \dot{\boldsymbol{\epsilon}}(t)-\frac{3}{\tau_{m}}(\operatorname{det} \mathbf{A})^{5 / 3} \mathbf{A} \operatorname{dev} \mathbf{G},
\end{aligned}
$$

Brittle and ductile material behavior. The aim of this test is not only the validation of the new ODE solver with exponential time integration, but also the capability of the GPR model with material failure to describe completely different material behavior with one and the same set of governing equations, just changing the model parameters appropriately. In particular, the proposed model is able to reproduce brittle material as well as ductile material behavior.

We set the strain rate to $\dot{\epsilon}_{11}(t)=-0.001 \mathrm{~s}^{-1}$ and compute the solution until a fixed end time $t_{\text {end }}=20 \mathrm{~s}$, first with the new exponential integrator with adaptive timestepping, and then with the scipy.integrate.solve_ivp Python library routine, which contains a wrapper to the popular LSODA/ODEPACK Fortran code [74]. Furthermore, we also show the solution obtained by integrating the governing ODE in $10^{4}$ uniform timesteps with the implicit Euler scheme.

In Figure 2 we plot the von Mises stress $Y=\sqrt{3 \operatorname{tr}(\operatorname{dev} \boldsymbol{\sigma} \operatorname{dev} \boldsymbol{\sigma}) / 2}$ against time, together with the time evolution of the damage variable $\xi$. In a first run (first column of Figure 2) the material parameters are chosen so that the material exhibits a characteristically brittle behavior (like ceramics or glass), with linear elastic deformation until the failure point (clearly marked by the jump in the damage variable $\xi$ ), while a second run is carried out with ductile material (like for example most metals). In this latter case (second column of Figure 2), one can clearly distinguish an initial linear elastic deformation, followed by a nonlinear transition into ideal plastic flow until eventual failure. Our example brittle material is obtained with the following choice of model parameters: $\rho_{0}=3000 \mathrm{~kg} \mathrm{~m}^{-1}, \mu_{I}=30 \mathrm{GPa}, \mu_{D}=30 \mathrm{MPa}, \lambda_{I}=\lambda_{D}=60 \mathrm{GPa}, \tau_{I 0}=3 \times 10^{3} \mathrm{~s}$, $\tau_{D 0}=3 \mathrm{~s}, \theta_{0}=8, a=32.5, Y_{0}=1.4 \mathrm{GPa}, Y_{1}=10 \mathrm{MPa}, \alpha_{I}=35, \alpha_{D}=35, \beta_{I}=2.2 \times 10^{-8} \mathrm{~Pa}^{-1}$, $\beta_{D}=2.2 \times 10^{-7} \mathrm{~Pa}^{-1}$, while the ductile behavior is given with $\rho_{0}=3000 \mathrm{~kg} \mathrm{~m}^{-1}, \mu_{I}=30 \mathrm{GPa}$, $\mu_{D}=30 \mathrm{MPa}, \lambda_{I}=\lambda_{D}=60 \mathrm{GPa}, \tau_{I 0}=1 \times 10^{3} \mathrm{~s}, \tau_{D 0}=1 \mathrm{~s}, \theta_{0}=1, a=1, Y_{0}=8 \mathrm{TPa}$, $Y_{1}=4 \mathrm{MPa}, \alpha_{I}=0, \alpha_{D}=30, \beta_{I}=2 \times 10^{-8} \mathrm{~Pa}^{-1}, \beta_{D}=1 \times 10^{-4} \mathrm{~Pa}^{-1}$.

The results from the exponential integrator are in perfect agreement with the LSODA reference solution, while it is apparent that $10^{4}$ timesteps with the implicit Euler method are not sufficient for adequately capturing the sudden onset of material failure.

Rate-dependent behavior and material fatigue. In this test we want to show that our model is a so-called rate-dependent model, that is, the maximum stress that can be sustained by the material before the failure point is reached, can vary as a function of the speed of deformation, i.e. the strain rate. It is quite common for example that impacts or explosions allow a given material to achieve a higher equivalent stress than slow (quasi-static) loads, before total breakdown.

To show that effects of this type can be reproduced by our model, we repeat the previous test on the ductile material, but by varying the strain rate from $\dot{\epsilon}_{11}(t)=-0.001 \mathrm{~s}^{-1}$ to $\dot{\epsilon}_{11}(t)=-0.002 \mathrm{~s}^{-1}$ and $\dot{\epsilon}_{11}(t)=-0.004 \mathrm{~s}^{-1}$, reaching higher maximum equivalent stress states as strain is applied faster. This is shown in Figure 3, where we also report the time evolution of the damage parameter $\xi$, with logarithmic scaling, in order to highlight that very small deviations of this variable from the perfectly intact state (of the order of $10^{-15}$ to $10^{-5}$ ) can indicate the difference between the Hookian elastic response and the plastic regime.

Finally, we want to show that the proposed model can also describe fatigue effects by allowing the material to retain memory of previous stress states. In fact, thermodynamical consistency prescribes that the source term associated with the damage variable always be positive (or possibly vanishing), this means that any stress applied to the material will ever so slightly damage it and 
eventually cause a deterioration of its mechanical properties. The extremely nonlinear response to different intensity levels of the stress norm determines that appropriate choices of parameters can describe materials with very late onset of fatigue effects, as well as properly governing the deterioration process following a certain level of exposure to cyclic stress.

For this test we employ an elasto-plastic material characterized by the following choice of parameters: $\rho_{0}=3000 \mathrm{~kg} \mathrm{~m}^{-1}, \mu_{I}=30 \mathrm{GPa}, \mu_{D}=33 \mathrm{MPa}, \lambda_{I}=\lambda_{D}=60 \mathrm{GPa}, \tau_{I 0}=2 \times 10^{5} \mathrm{~s}$, $\tau_{D 0}=2 \times 10^{3} \mathrm{~s}, \theta_{0}=1, a=1, Y_{0}=8 \mathrm{TPa}, Y_{1}=8 \mathrm{TPa}, \alpha_{I}=0, \alpha_{D}=0, \beta_{I}=3 \times 10^{-8} \mathrm{~Pa}^{-1}$,
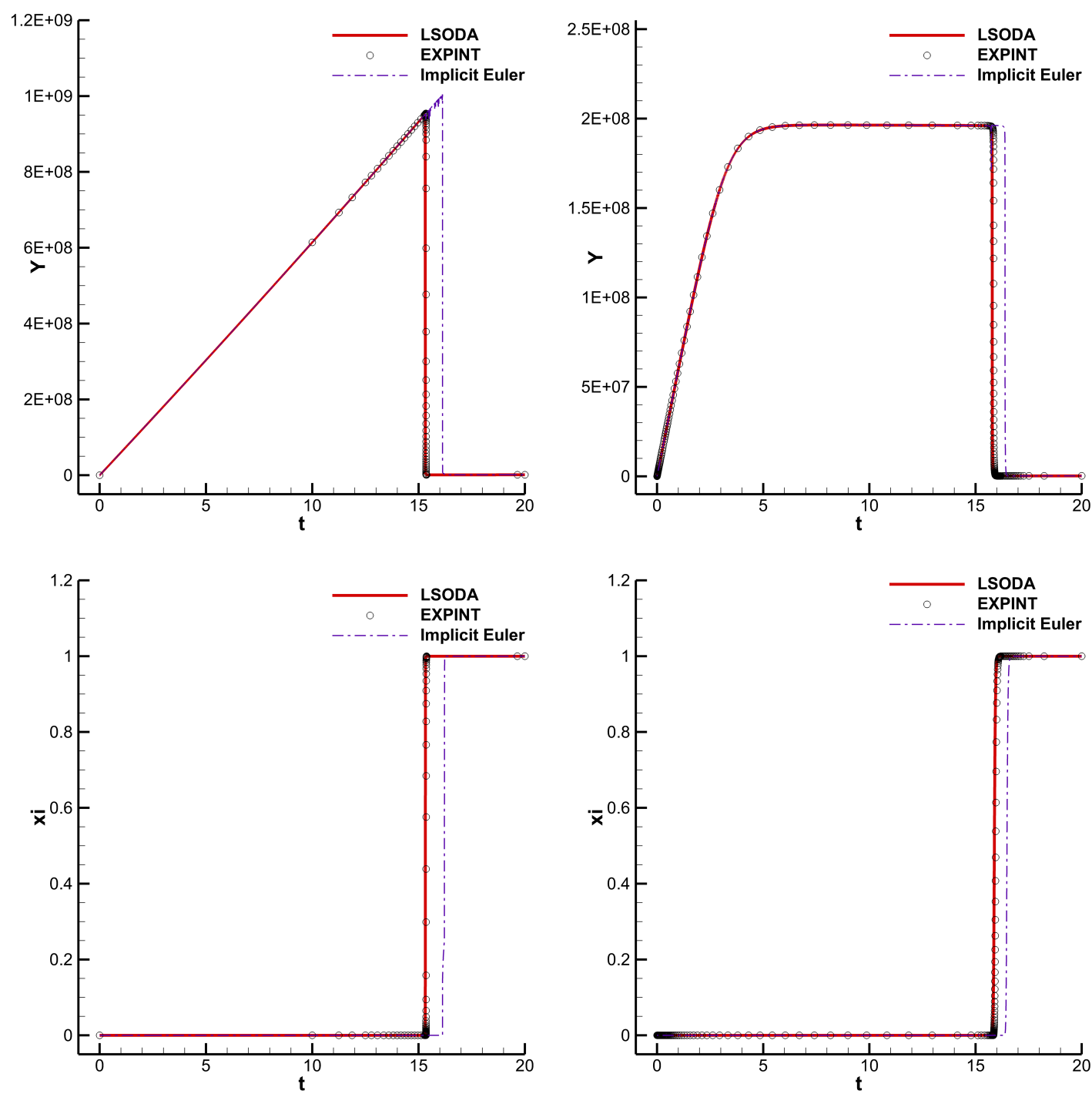

Figure 2: Stress-strain diagrams (top row) and time evolution of the damage variable $\xi$ (bottom row) for an example material characterized by brittle failure (left column) and for a material with ductile failure mode (right column). We show that the solution obtained from the proposed exponential integration method matches the reference given by the LSODA library, while integration with the simple implicit Euler method can yield inaccurate solutions even with very small timesteps. 
$\beta_{D}=0 \mathrm{~Pa}^{-1}$. We setup a series of computations by first subjecting the material to a low intensity cyclic stress (about $20 \%$ of the limit for the elastic regime), determined by a variable strain rate of the form $\dot{\epsilon}_{11}(t)=-0.001 \sin (2 \pi t) \mathrm{s}^{-1}$, and then measuring the residual strength of the material by means of a quasi-static destructive test as those shown in Figures 2 and 3 . A plot of the material distortion caused by this type of forcing (low intensity periodic deformations followed by a destructive test) is shown in Figure 4, together with the numerical stress-strain diagram of an initially intact sample under slow (quasi-static) loading. The main result of this benchmark is the so-called Wöhler diagram: for this purpose, in the top left panel of Figure 4 we show a bilogarithmic plot of the material strength as a function of the number of applied cycles. The Wöhler diagram obtained in our simulations shows the same qualitative behaviour as those obtained in experiments for real materials, with the classical division into three different regimes: short, intermediate and long-term durability. Our numerical results illustrate that the test material shows no signs of weakening at a low number of load cycles (up to about 1000). For more load cycles, one can note the typical decay of the material resistance, while for a very large number of cycles the the long-time durability limit is reached, which, however, still shows a small but steady degradation, as it is commonly found in aluminum-alloys. In order to give the reader a more detailed insight about how fatigue is accounted for in our model, we also show the temporal evolution of the damage variable $\xi$ (bottom right panel) for three of the tests (one at $N=1000$ elastic cycles, one at $N=5000$ cycles, one at $N=10000$ cycles), showing that the accumulation of fatigue effects is well captured by the exponential growth of the damage variable.

At this point we would like to emphasize that the addition of the thermodynamically compatible evolution equation (1f) of a simple scalar $\xi$ to the GPR model $[96,47]$ is enough to model such a complex behaviour as material fatigue.
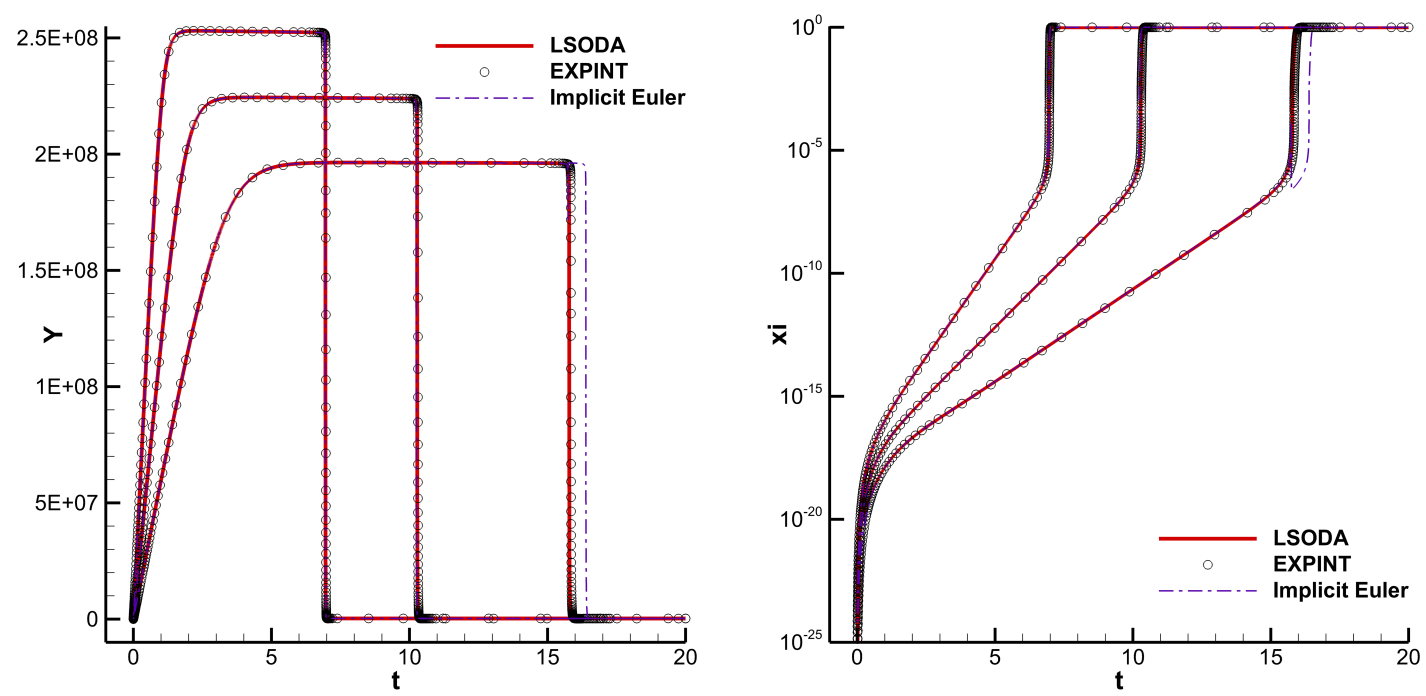

Figure 3: Rate-dependent stress-strain diagrams (left panel) and time evolution of the damage variable (right panel) for a ductile material subjected to three different constant strain rates. 

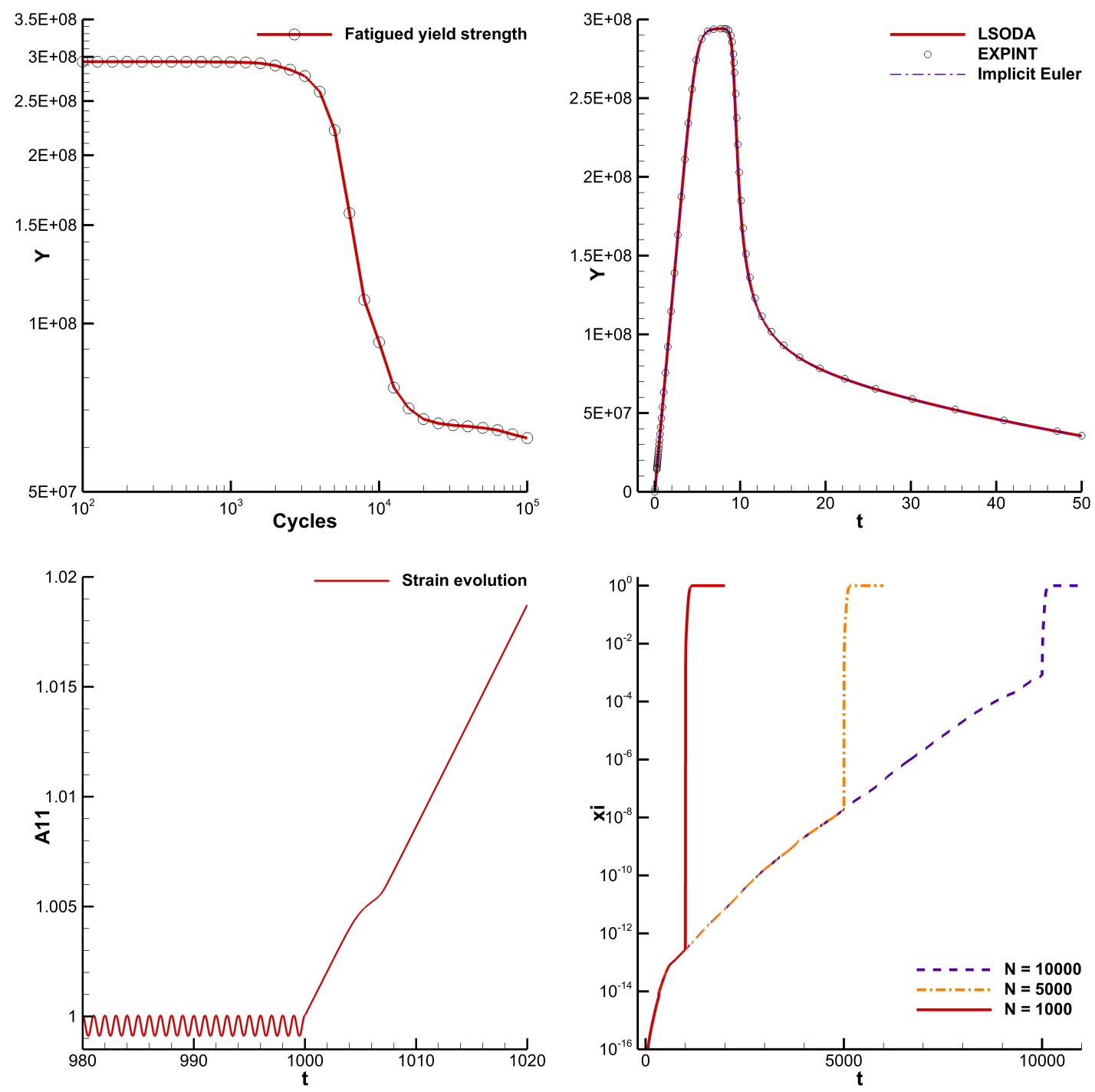

Figure 4: Numerical results obtained for the fatigue behaviour. Top left panel: Wöhler diagram, showing the weakening of the material strength as a function of the number of applied load cycles. Top right panel: stress-strain diagram of the test material for a single, quasi-static (slow) load cycle until rupture. Bottom left panel: evolution of the $A_{11}$ component of the distortion field for the last elastic load cycles in the case $N=1000$ and final traction test until material failure. Bottom right panel: temporal evolution of the damage variable $\xi$ for three different numbers of load cycles $N$. 


\section{Numerical results}

If not stated otherwise, throughout the entire section all units are in SI units. In the case of adaptive mesh refinement, the AMR refinement factor between two adjacent grid levels is always $\mathfrak{r}=3$, see $[51,132,101]$ for details on AMR.

\subsection{Stiff inclusion test case}

The first test that we show here was originally proposed by LeVeque [80] in Chapter 22.7 for linear elasticity. The test consists in a p-wave that travels through a heterogeneous medium with free surface boundary conditions on the top and bottom boundaries of the computational domain. In the original test, an outer solid medium $\Omega_{\text {out }}=[-1,1] \times[-0.5,0.5]$ contains another stiff material placed in $\Omega_{i n}=[-0.5,0.5] \times[-0.1,0.1]$. The Lamé parameters for the two materials are $(\lambda, \mu, \rho)_{\text {out }}=(2,1,1)$ and $(\lambda, \mu, \rho)_{\text {out }}=(200,100,1)$ so that the resulting $\mathrm{p}$ - and s- wave speeds in the stiffer material are 10 times larger than in the other material.

The main purpose of this test problem in the context of nonlinear hyperelasticity is to verify if the numerical method generates spurious pressure oscillations at the moving interface where the Lamé parameters jump by two orders of magnitude, or not. It is well known from conservative numerical methods applied to the compressible Euler equations that spurious pressure oscillations are generated when solving the Euler equations with a spatially variable ratio of specific heats, see e.g. $[121,2]$ for a more detailed discussion. Note that in our model the PDE for the transport of the Lamé constants and the parameter $Y_{0}$ is written in non-conservative form, see eqn. (2).

The initial condition for the travelling $\mathrm{p}$-wave is given by

$$
\begin{aligned}
\boldsymbol{\sigma} & =\epsilon R_{\sigma} \cdot \exp \left(-\frac{1}{2} \frac{\left(x-x_{0}\right)^{2}}{\delta^{2}}\right) \\
\mathbf{v} & =\epsilon R_{\mathbf{v}} \cdot \exp \left(-\frac{1}{2} \frac{\left(x-x_{0}\right)^{2}}{\delta^{2}}\right)
\end{aligned}
$$

where $\boldsymbol{\sigma}=\left(\sigma_{x x}, \sigma_{y y}, \sigma_{z z}, \sigma_{x y}, \sigma_{y z}, \sigma_{x z}\right), R_{\sigma}=(4,2,2,0,0,0), R_{\mathbf{v}}=(-2,0,0), x_{0}=-0.8$ and $\delta=0.01$ represents a p-wave of Gaussian shape travelling in the $x$-direction. Since the test regards a linear elastic medium, we chose $\epsilon=10^{-4}$ in order to have small deformations and negligible

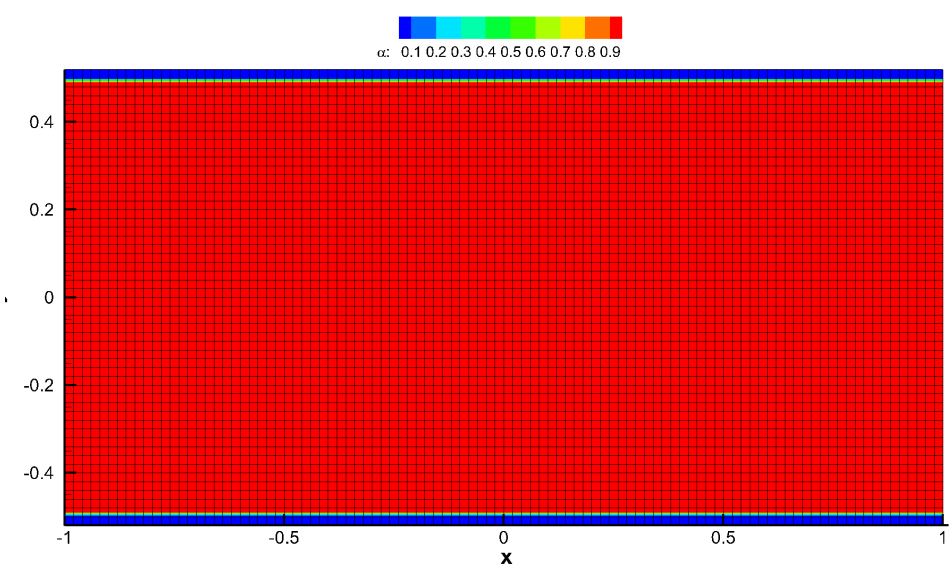

Figure 5: Shape of the diffuse interface, represented by the solid volume fraction function $\alpha$. 
contributions from the nonlinear convective part. The initial condition for the matrix $\mathbf{A}$ is obtained by fixing a direction angle $\theta=0$ and then use the procedure $\mathbf{A}=\mathbf{A}(\boldsymbol{\sigma}, \theta)$ as described in Appendix A. The thermal impulse is set to $\mathbf{J}=0$ and furthermore in this test we set $c_{h}=0$.

Finally the free surface boundary conditions are obtained by enlarging the computational domain to $\Omega=[-1.0,1.0] \times[-0.52,0.52]$ and then use the diffuse interface parameter $\alpha$ in order to identify the location of the solid medium, i.e. setting $\alpha=1$ for $\mathbf{x} \in \Omega_{\text {out }}$ and $\alpha=0$ elsewhere. In order to prevent spontaneous ruptures, both materials are assumed to be unbreakable, i.e. the yield stress is set to the very large value $Y_{0}=10^{22} \mathrm{~Pa}$. The domain is covered using $100 \times 52$ elements and we use fourth order polynomials $N=M=4$ in space and time.

The inclusion of the constraint $\rho / \rho_{0}=|A|$ is crucial in order to obtain a solution due to the heterogeneity of the medium.

The resulting profile of the diffuse interface $\alpha$ is reported in Fig. 5, while the time evolution of the stress component $\sigma_{x x}$ is reported in Fig. 6. In Fig. 6 one clearly can observe the influence of the free surface in the propagation of the p-wave, before it hits the stiff inclusion. Note that in our diffuse interface approach, the free surface boundary condition is fully taken into account by the governing PDE system and the scalar volume fraction function $\alpha$. No explicit boundary conditions need to be applied, see also [115]. Then the pattern generated after the hit with the stiff material results very similar to the one obtained in [78] with an ADER-DG scheme of fourth order of accuracy on a boundary-fitted mesh of $4 \cdot 200 \times 100$ triangular elements.

From the obtained computational results one clearly observes that the produced wavefield is free from spurious pressure oscillations at the interface and that therefore also jumps in the Lamé constants by two orders of magnitude can be properly handled by our numerical method.
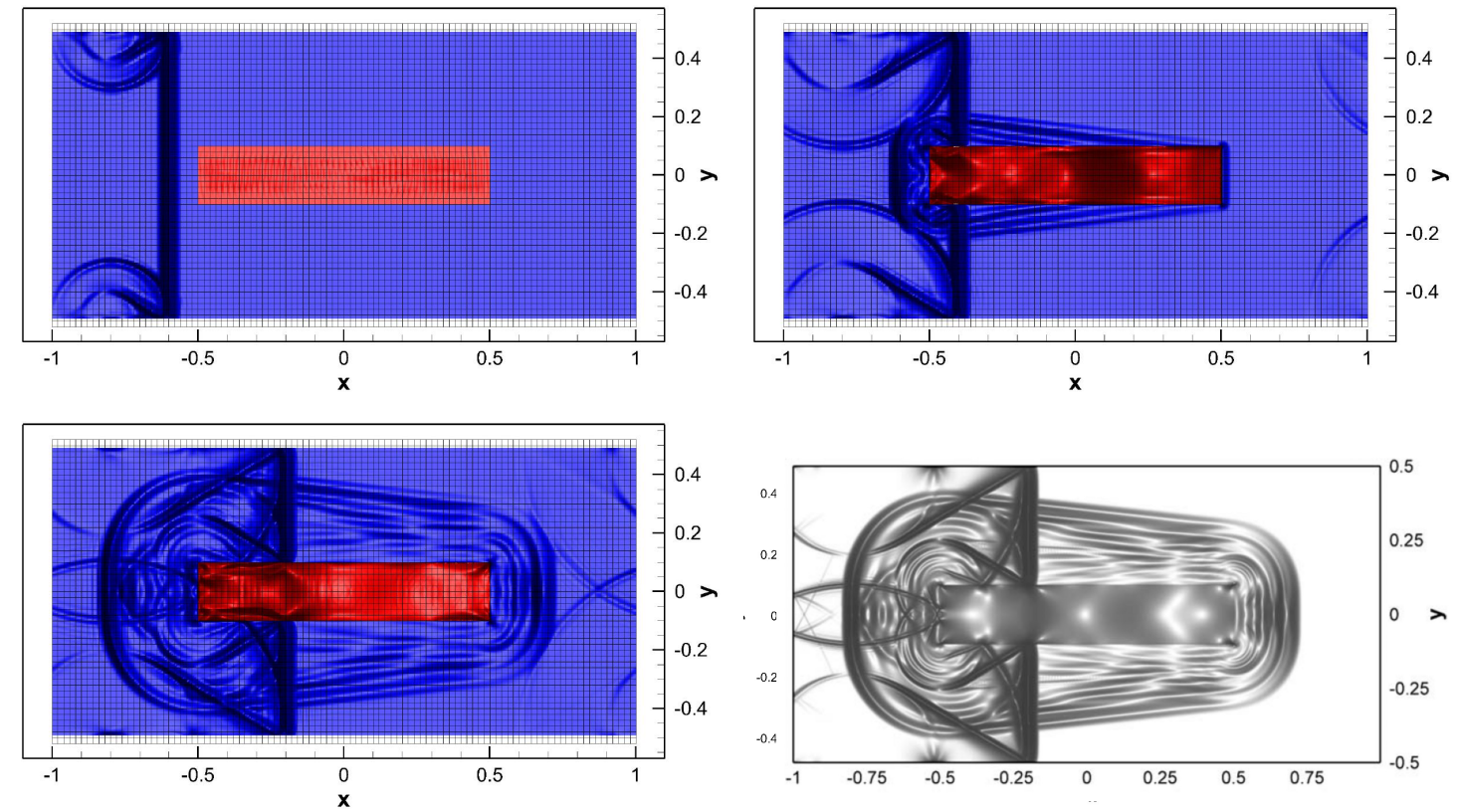

Figure 6: Evolution of the stress component $\sigma_{x x}$ at times $t=0.1,0.2$ and $t_{\text {end }}=0.3$ colored with $\lambda$. In the bottom right figure we report the numerical solution obtained in [78] with an ADER-DG scheme of fourth order on $4 \cdot 200 \times 100$ triangular elements. 


\subsection{Fracture generation after high speed impact of a copper plate onto a pyrex glass}

Here we want to study the wave and rupture propagation driven by the impact of a flying copper plate that impacts on a Pyrex glass brick, following the setup proposed in [103]. We consider first a $1 D$ impact problem of a copper plate onto a pyrex glass. A sketch of the experimental setup is depicted in Fig. 7. As discussed in [103], a proper equivalent stress $Y$ has to include both shear and pressure contributions. For this numerical experiment we use the following linear definition of the equivalent stress:

$$
Y=A Y_{s}+B\left|Y_{p}\right|
$$

where, $Y_{s}=\sqrt{3 \operatorname{tr}(\operatorname{dev} \boldsymbol{\sigma} \operatorname{dev} \boldsymbol{\sigma}) / 2}$ is the von Mises shear stress norm, $Y_{p}=\operatorname{tr} \boldsymbol{\Sigma} / 3$ accounts for the pressure contribution, and the weighting factors are set to $A=0.9$ and $B=0.05$.

As numerical parameters we take a computational domain $\Omega=[-0.001,0.021] \times[-0.001,0.001]$ covered with $108 \times 8$ elements, polynomial approximation degree $N=M=3$ and periodic boundaries in the $y$-direction. We use a multi-material approach where the mechanical properties inside the domain are set as unstressed Pyrex, while copper is located at the left boundary (see Table 1 for material parameters), where also an impact velocity $v_{B}$ is assumed. For this test we use two different impact velocities of $v_{1}=250 \mathrm{~m} / \mathrm{s}$ and $v_{2}=530 \mathrm{~m} / \mathrm{s}$, as done in the experimental setup, see [22]. We take the time series of the stress, computed as $\sigma=\left|\sigma_{x x}\right|$, in $x=2.5 \mathrm{~mm}$.

The comparison between numerical and experimental stress, properly shifted according to the $p$-wave velocity, is reported in Figure 8. Note here that the use of multi-material is crucial in order to obtain the proper velocity inside the target object. The resulting penetration velocity for the two cases are, respectively $v_{p, 1} \approx 426 \mathrm{~m} / \mathrm{s}$ and $v_{p, 1} \approx 187 \mathrm{~m} / \mathrm{s}$ and strictly depend on the mechanical properties of the bullet. We now move to a two dimensional experiment where a hemispherical projectile hits a Pyrex glass block. According to [103] we need to generalize the equivalent stress

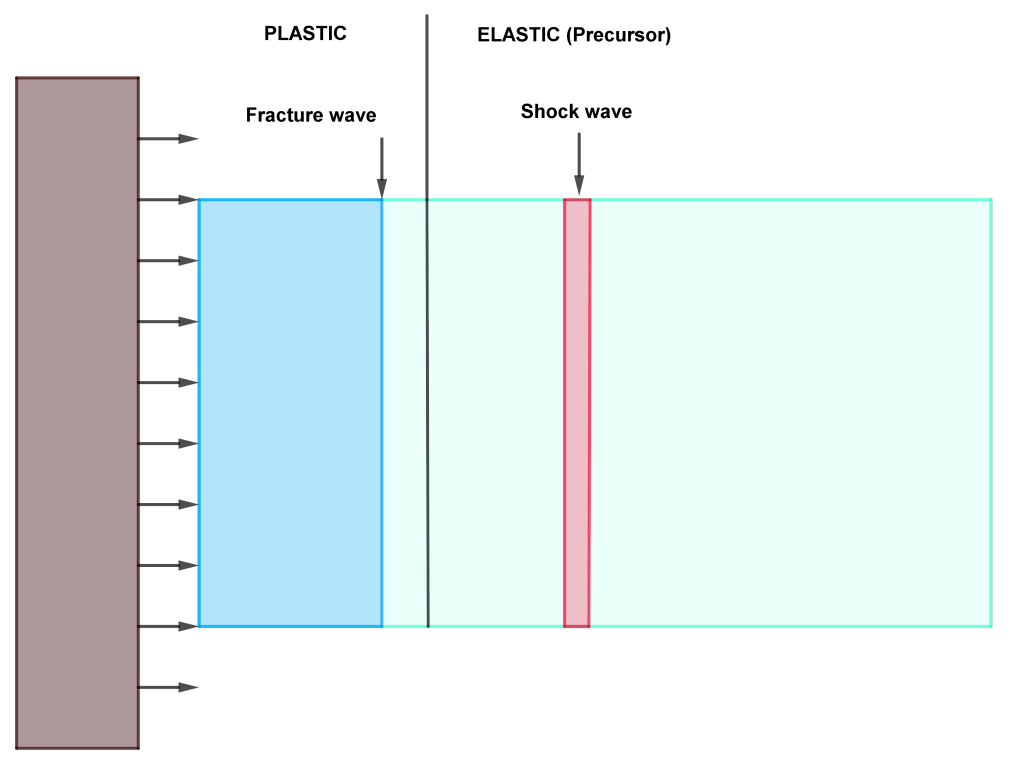

Figure 7: Sketch of the experimental setup. 
definition as follows

$$
Y=B\left|Y_{p}\right|+A \frac{1}{2}\left(Y_{s}-s_{0}\right)\left[1+\operatorname{erf}\left(\frac{Y_{s}-s_{0}}{\epsilon}\right)\right],
$$

where $\epsilon=10^{-3} Y_{0}$ is a smoothing factor and $s_{0}=1.8 \mathrm{GPa}, A=0.3$ and $B=0.33$. The computational domain is taken as $\Omega=[-0.005,0.005] \times[-0.0104,0.0004]$ covered with $50 \times 54$ elements and using a polynomial approximation degree of $N=M=2$. The hemispherical projectile is represented through time dependent boundary conditions. The impact velocity is set as $v=536 \mathrm{~m} / \mathrm{s}$, while the penetration velocity, that here changes the impact area, is extrapolated from [103] where also the bullet dynamics was considered. The resulting evolution of the damaged front is compared with the experimental pictures taken by Bourne in 1997, see [21], and is reported in Figure 9. The damage front propagation is close to the one obtained in the experiments and is slower than the elastic precursor wave as shown in Figure 10. The evolution of the damage variable $\xi$ is presented in Figure 10, showing the evolution of the fracture wave in the Pyrex block. Furthermore, secondary cracks can be observed in Figure 9, similarly to the one obtained in [103]. The time evolution of the bullet penetration is reported in Fig. 11 where the rupture front is also depicted. Note that the damage variable $\xi$ is transported during the bullet impact (blue area) and no rupture in the copper is observed due to the strongest material properties. We can finally observe how for long times the shape of the rupture is different in the numerical case and is more close to the case of a flat-nose rod, see again [103]. Nevertheless, the experimentally observed propagation velocity of the rupture front is very well reproduced in our numerical simulations.

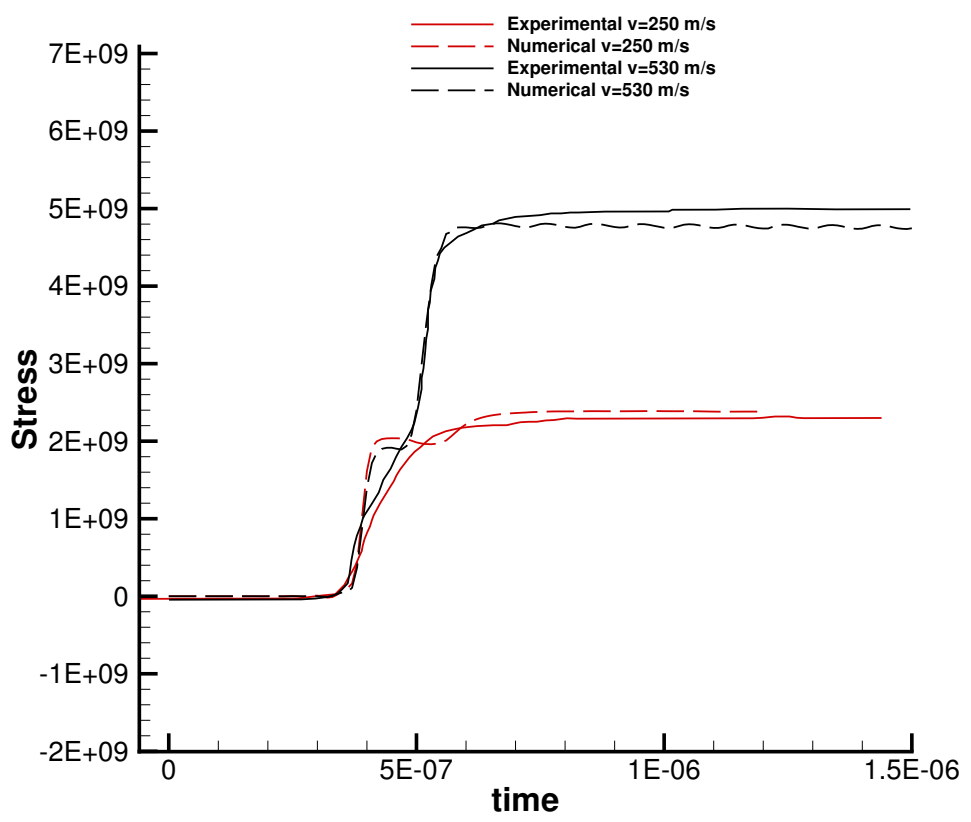

Figure 8: Time series of the computed stress defined as $\sigma=\left|\sigma_{x x}\right|$ compared with experimental results [103, 22] for two different impact velocities, namely $v_{1}=250 \mathrm{~m} / \mathrm{s}$ and $v_{2}=530 \mathrm{~m} / \mathrm{s}$. 

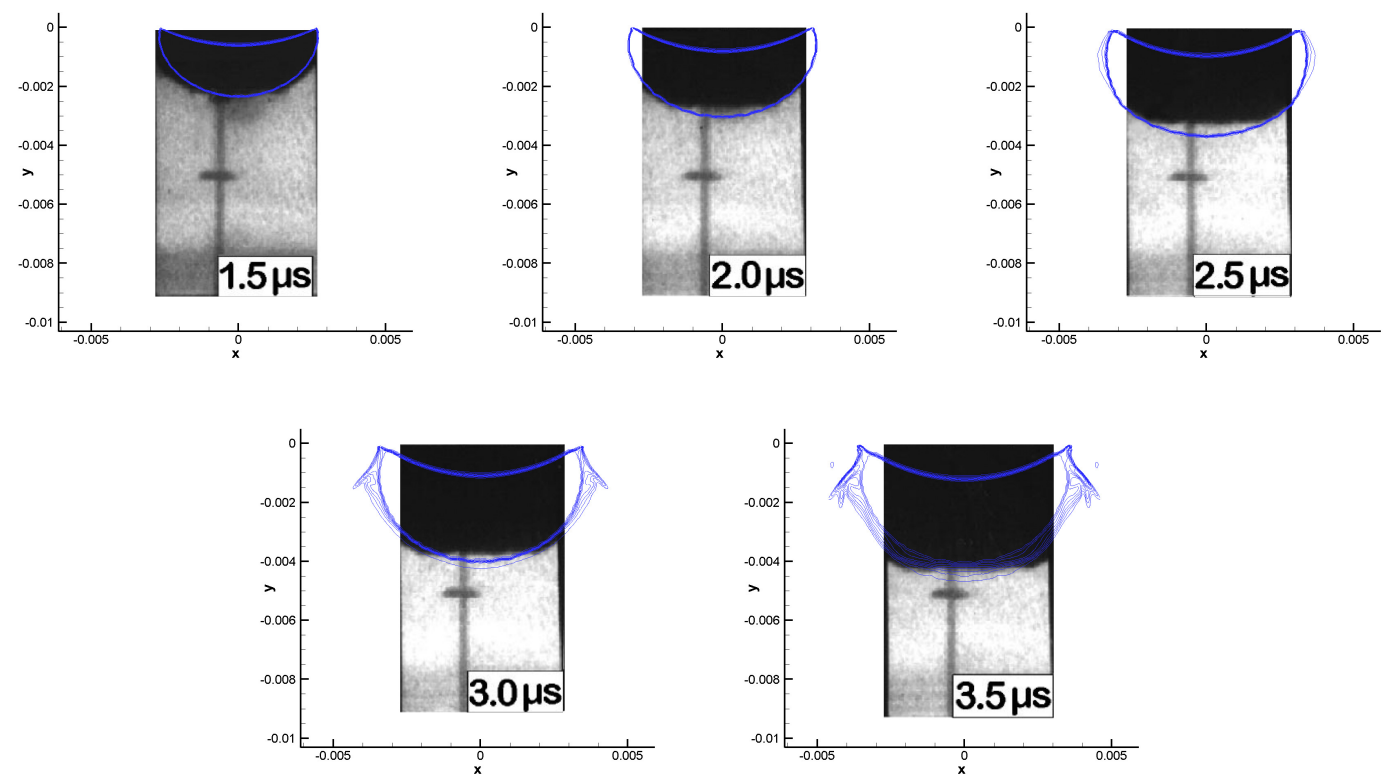

Figure 9: Comparison between numerical fracture front (blue lines) with experimental results at several times.
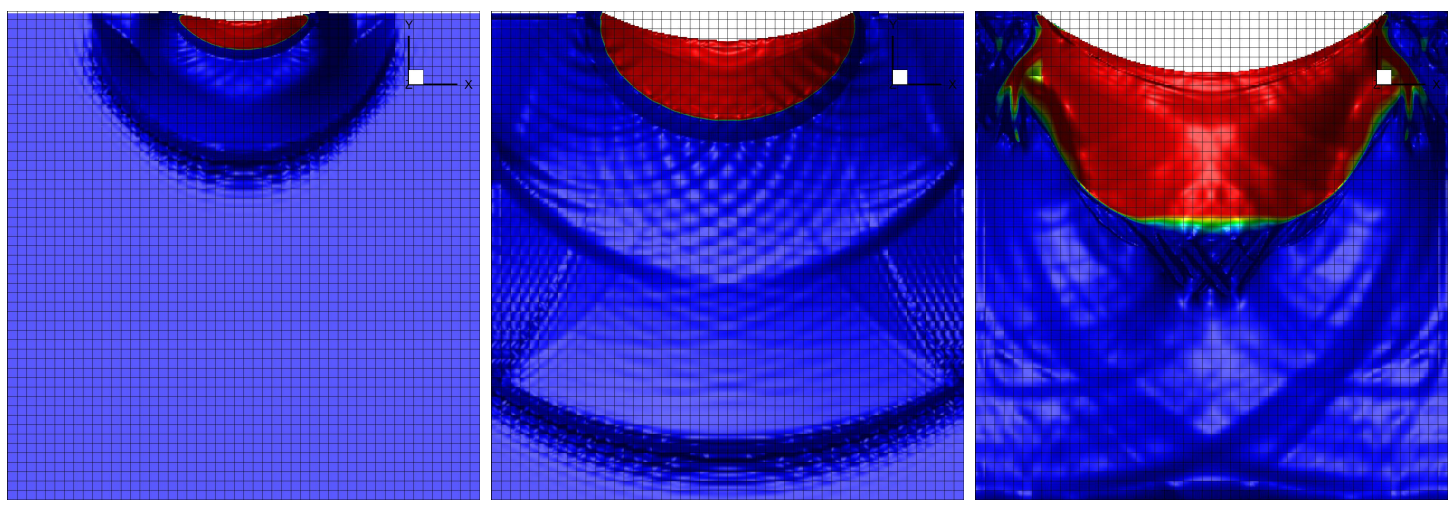

Figure 10: Two dimensional crack propagation and wave pattern at times $t=0.5,1.5$ and $3.8 \mu \mathrm{s}$. 

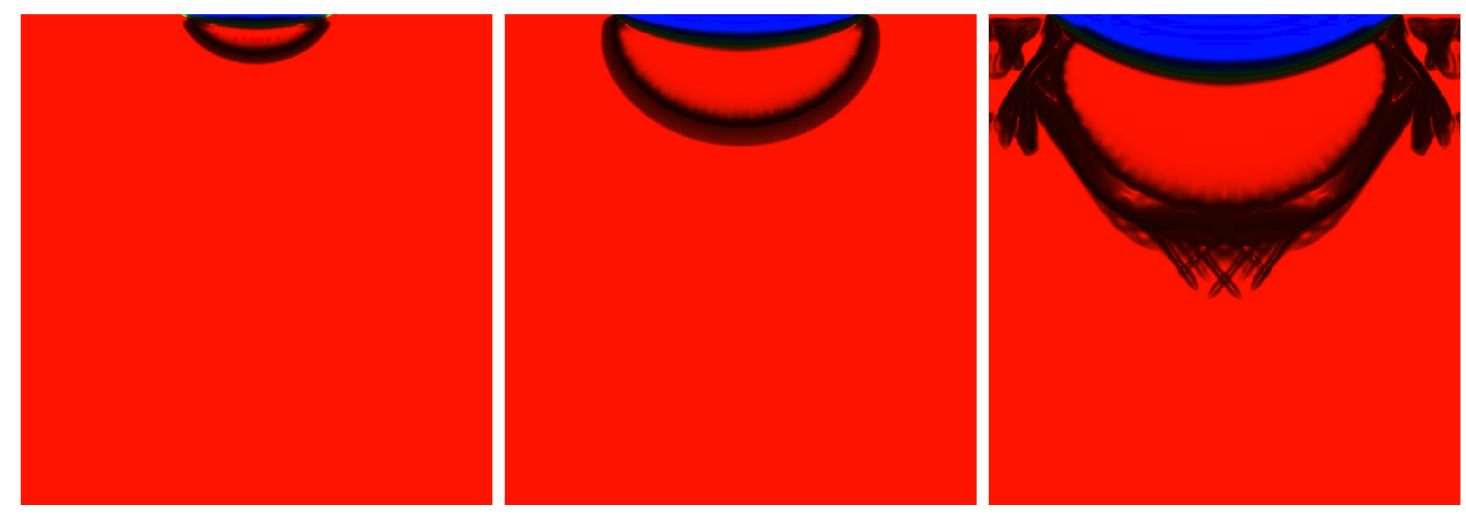

Figure 11: Penetration pattern of the unbreakable copper (blue zone) in the Pyrex material (red zone) at times $t=0.5,1.5$ and $3.8 \mu \mathrm{s}$. The evolution of the rupture front is also reported (shadow zone) and shows the transport of the rupture parameter according to the bullet penetration.

Table 1: Set of parameters for the materials.

\begin{tabular}{lllllll}
\hline & Rock 1 & Rock 2 & Rock 3 & Rock 4 & Pyrex & Copper \\
\hline$\rho\left[\mathrm{kg} \mathrm{m}^{-3}\right]$ & 2670 & 2620 & 2670 & 2670 & 2230 & 8930 \\
$\mu_{I}[\mathrm{GPa}]$ & 32.04 & 21.44 & 32.04 & 32.04 & 30.36 & 48.27 \\
$\mu_{D}[\mathrm{GPa}]$ & 27.46 & 0.15008 & 27.46 & 0.03204 & 0.1518 & 41.38 \\
$\lambda_{I}[\mathrm{GPa}]$ & 32.04 & 21.44 & 32.04 & 32.04 & 20.90 & 105.79 \\
$\lambda_{D}[\mathrm{GPa}]$ & 35.10 & 0.15008 & 35.10 & 53.38 & 30.97 & 110.39 \\
$\theta_{0}[-]$ & 10.0 & 1.0 & 0.2 & 1.0 & 1.0 & 0.0 \\
$Y_{0}[\mathrm{MPa}]$ & 180 & 10 & 240 & 9.0 & 1200 & $10^{16}$ \\
$Y_{1}[\mathrm{MPa}]$ & 10.0 & $10^{-6}$ & 10.0 & 10.0 & 10.0 & $10^{16}$ \\
$a[-]$ & 42.5 & 60.0 & 42.5 & 52.5 & 32.5 & 1.0 \\
$\alpha_{I}[-]$ & 36.25 & 0.0 & 36.25 & 36.25 & 36.25 & 40.0 \\
$\alpha_{D}[-]$ & 36.25 & 0.0 & 36.25 & 36.25 & 34.8 & 40.0 \\
$\beta_{I}\left[\mathrm{~Pa}{ }^{-1}\right]$ & 0.0 & 0.0 & 0.0 & 0.0 & $22.31 \times 10^{-9}$ & 0.0 \\
$\beta_{D}[\mathrm{~Pa}-1]$ & $10^{-6}$ & 0.0 & $10^{-6}$ & $10^{-6}$ & $223.07 \times 10^{-9}$ & 0.0 \\
$\tau_{I 0}[\mathrm{~s}]$ & $3.2 \times 10^{6}$ & $10^{5}$ & $3.2 \times 10^{6}$ & $3.2 \times 10^{6}$ & $3.0 \times 10^{6}$ & $4.8 \times 10^{6}$ \\
$\tau_{D 0}[\mathrm{~s}]$ & $2.75 \times 10^{6}$ & $10^{-3}$ & $2.75 \times 10^{6}$ & $3.2 \times 10^{3}$ & $1.5 \times 10^{4}$ & $4.1 \times 10^{6}$ \\
\hline
\end{tabular}




\subsection{Fracture generation in a piece of pre-damaged rock}

In order to show the applicability of our new algorithm to realistic experiments that are frequently carried out in civil engineering and in geophysics, we consider the crack propagation in a pre-damaged rock-like disc (so-called Brazilian test), with the aim of comparing to the experimetal results given in [68]. The disc is described with the moving diffuse interface approach, and its boundary is thus represented by means of the solid volume fraction $\alpha$. An inclined slit is represented as predamaged zone. In order to match the experimental data, we rotated the disc with respect to the clamping apparatus in such a way that the slit has an inclination angle of $35^{\circ}$ with respect to the $x$-axis. We impose a velocity pointing towards the disc on the upper and bottom boundary, with a magnitude given by the gaussian profile $|v|=4 \exp \left(-(25 x)^{2}\right)$. We take our domain to be $\Omega=[-1.1,1.1]^{2}$ and employ a fourth order ADER-DG scheme with a-posteriori subcell limiting. The distribution of the volume fraction $\alpha$ of the diffuse interface method is depicted in Fig. 12. The material properties for the disc are those associated to Rock 2 in Table 1, while in the top and bottom clamps the material is modified by setting $Y_{0}=Y_{1}=100 \mathrm{TPa}$, effectively rendering these regions unbreakable. For the equivalent stress we take a Drucker-Prager-like combination of shear stress and pressure defined as

$$
Y=A\left|Y_{s}\right|+B Y_{p}+C
$$

where $A=1.0, B=1.5$ and $C=-2.0 \mathrm{MPa}$ The resulting evolution of the rupture is shown in Fig. 13. The main crack propagates starting from the corners of the pre-damaged zone, following the experimental results very closely to the top and bottom of the disc. In agreement with the experimental observations, we have also a damaged zone due to shear on the upper and lower part of the disc, while the rupture inside is driven by traction. Last but not least, we add a careful mesh

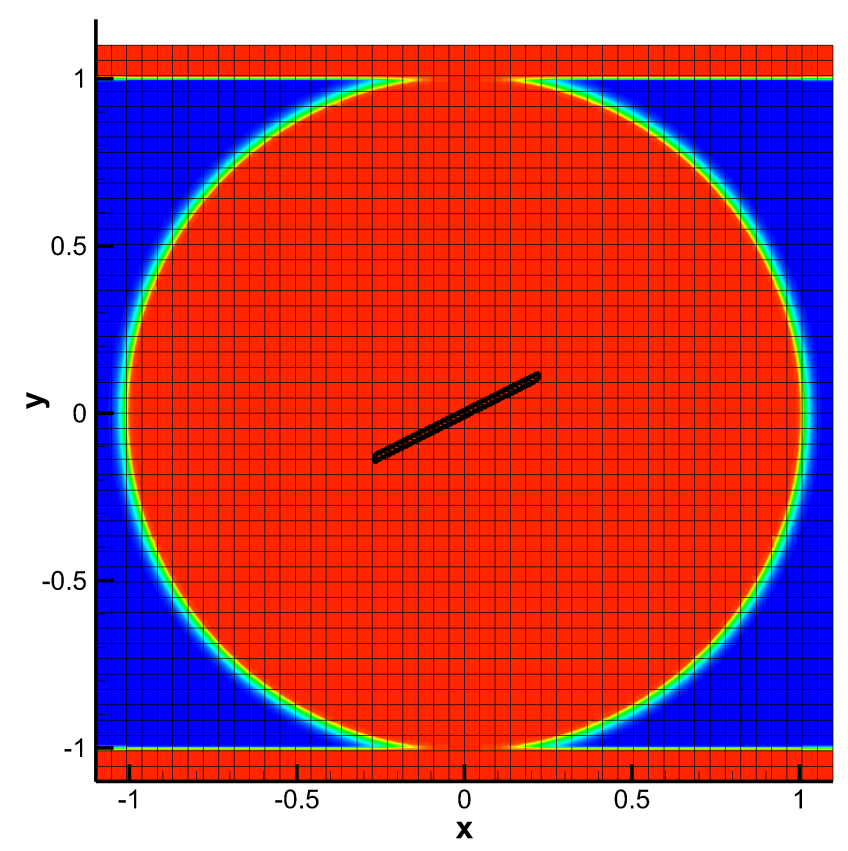

Figure 12: Representation of the pre-damaged disc in the diffuse interface approach via the solid volume fraction. The darkest area shows the pre-damaged zone. 
convergence analysis for this nontrivial test problem, in order to check whether the position and shape of the crack converges with mesh refinement. The result of this detailed mesh refinement study carried out on a sequence of uniform Cartesian grids with mesh resolution from $48 \times 48$ elements to $256 \times 256$ elements is shown in Figure 14 . One can observe that the main cracks properly converge with mesh refinement, in particular the crack becomes thinner on finer meshes. Only the secondary cracks generated at the upper and lower boundaries of the disc differ between one mesh and the other.

\subsection{Out of plane rupture with secondary and tertiary crack generation}

Here we consider the generation and propagation of a crack in a two dimensional domain $\Omega=[-5000,5000] \times[-7500,7500]$. The material is pre-damaged on the line $|x|<1000,|y|<66$, which is mesh aligned and that is loaded with an initial stress of $\sigma_{y y}=-120 \mathrm{MPa}$ and $\sigma_{x y}=$ $70 \mathrm{MPa}$. Here we simply use the von Mises stress for the definition of the equivalent stress. The considered material is ROCK 1 (see Table 1 for the defining parameters). In the numerical simulation we consider two AMR refinement levels that are activated dynamically according to the rupture propagation and that are needed to capture the small-scale structures that appear during the dynamic rupture process. We use a polynomial approximation degree of $N=M=3$ and on the coarsest level we consider two different meshes, a coarse one with $30 \times 24$ elements and a finer one
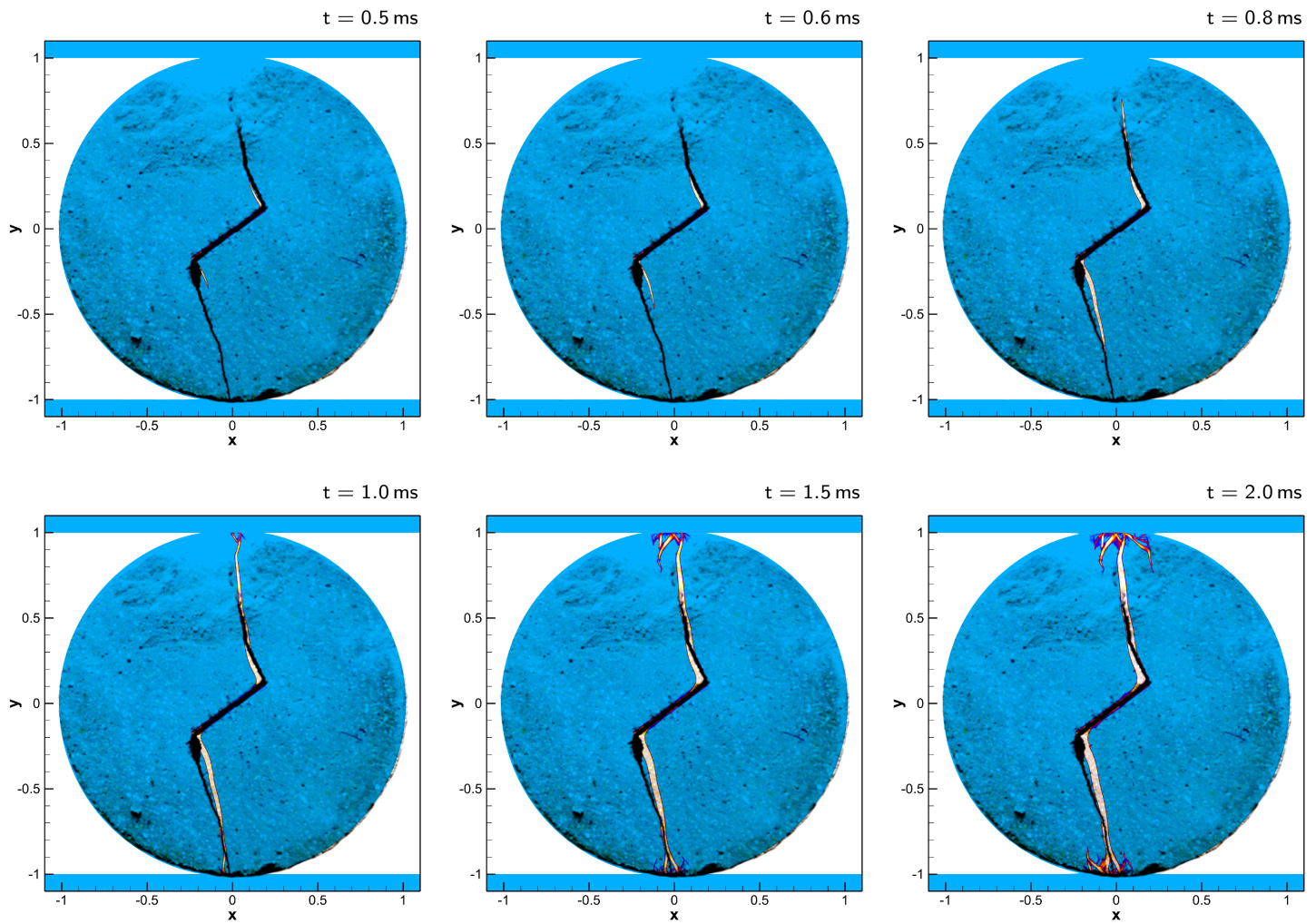

Figure 13: Evolution of the rupture front (color contours of the damage field $\xi$ ) compared with experiments at times $t=[0.5,0.6,0.8,1.0,1.5,2.0] \mathrm{ms}$. The computations are carried out with a fourth order ADER-DG scheme with a-posteriori subcell limiting on a uniform Cartesian grid of $256 \times 256$ elements. Only regions with $\alpha>0.5$ are shown. The experimental picture is taken from [68]. 
$48 \times 48$

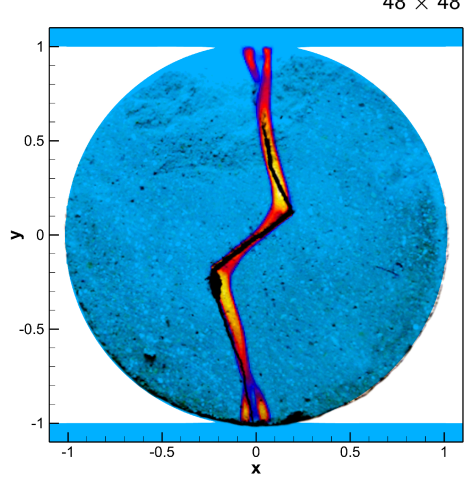

$128 \times 128$

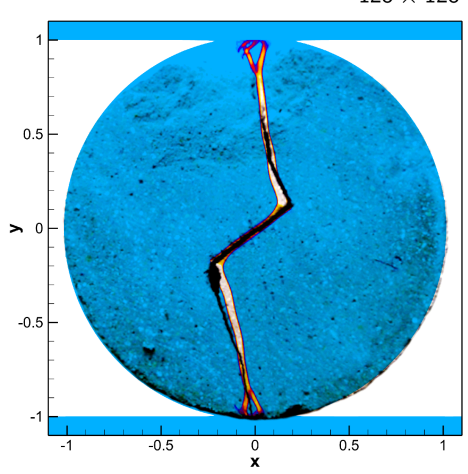

$64 \times 64$

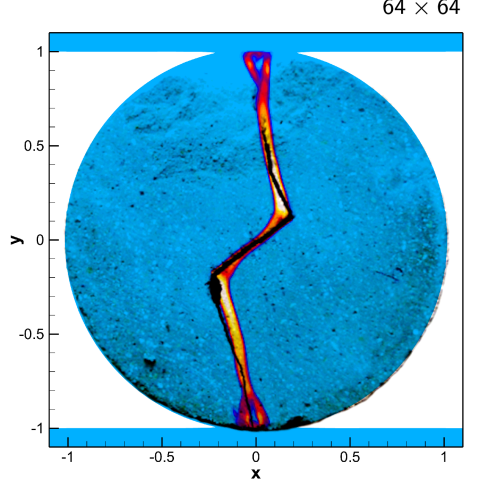

$192 \times 192$

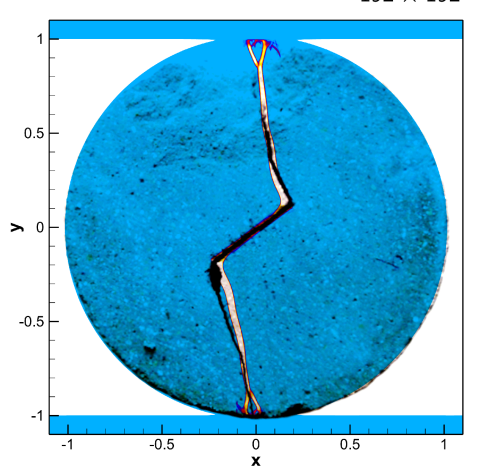

$96 \times 96$

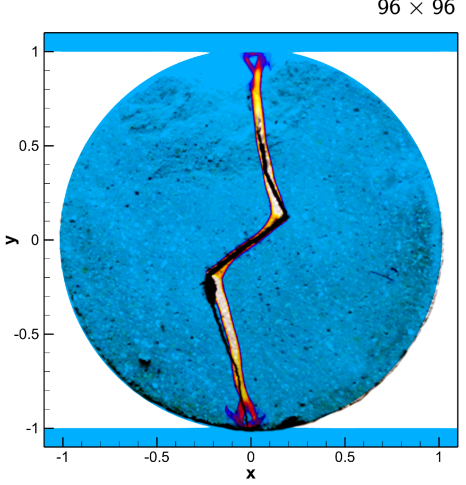

$256 \times 256$

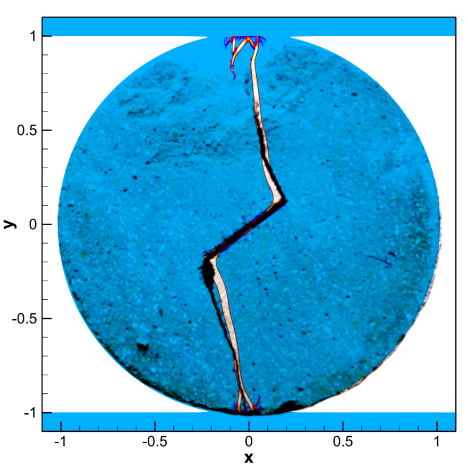

Figure 14: Mesh convergence study (color contours of the damage field $\xi$ ) for the simulation of a pre-damaged rock disc. The computations employ a fourth order ADER-DG scheme with MUSCL-Hancock a posteriori subcell finite volume limiting and show mesh convergence of the main cracks for six grid spacings ranging from $48 \times 48$ to $256 \times 256$ Cartesian cells. Only regions with $\alpha>0.5$ are shown. The experimental picture has been taken from [68]. 
with twice the resolution in each space dimension. The subcell FV limiter is activated according to [52], as using an additional physical detection criterion based on the ratio of the von Mises stress and the yield stress of the material. A comparison between the crack propagation using the coarse and the fine mesh at time $t=5.0 \mathrm{~s}$ is reported in Figure 15. Here we can observe how the direction of the main crack is the same for both grid resolutions, while with higher resolution we generate also several secondary and tertiary cracks that bifurcate from the main one. This is the expected behaviour due to the better resolution of shear bands forming. In this test one secondary crack prevents energy accumulation close to the corner points, see top right panel in Figure 15.

In order to show the effect of heterogeneous materials in the crack propagation we consider the same domain using $64 \times 100$ elements with one AMR refinement level and two different materials, namely ROCK 1 and ROCK 3, that are characterized by a different yield stress. The resulting time evolution of crack lines at times $t=[0.5,0.9,5.0]$ are reported in Figure 16, where also the horizontal velocity component is depicted. We can note how the presence of the second material, whose interface is represented by the black line in 16, breaks the symmetry of the rupture propagation that is decelerated in the ROCK 3. Furthermore, the secondary cracks seem to naturally align with the interface between the two materials. Finally, the thermal trace is reported in 17 and shows the energy that is converted into thermal energy due to friction and localized on the sliding lines.
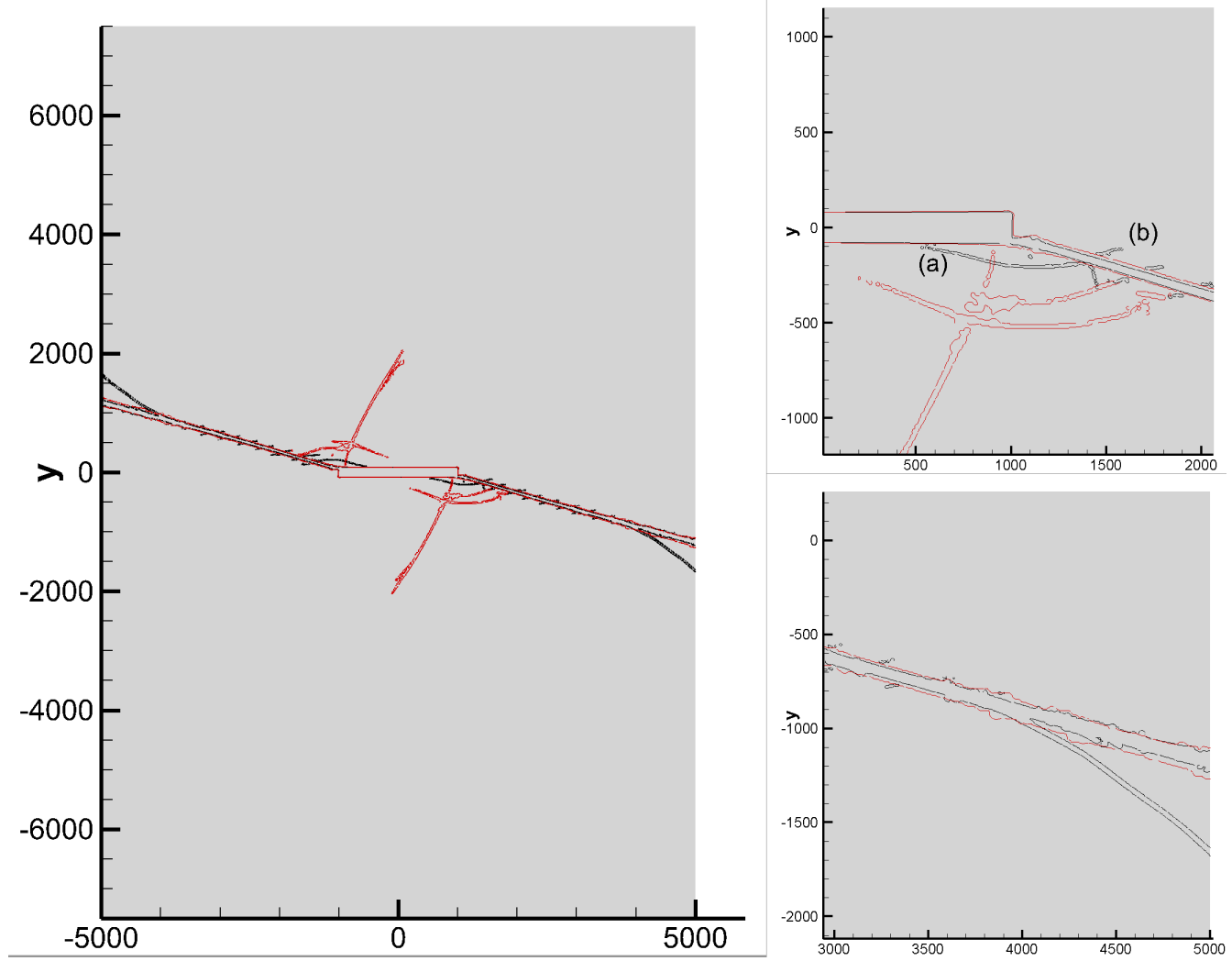

Figure 15: Out of plane crack propagation. Comparison of the crack profile for a coarse mesh (red line) and a finer one (dark line) at $t=5.0 \mathrm{~s}$. The main crack direction is the same, independently from the resolution, while small secondary cracks are more present on the finer mesh (b). 


\subsection{Double coin crack in three space dimensions}

We can obviously apply the same algorithm also in three space dimensions. Also in this case we take advantage of dynamic adaptive mesh refinement and the a posteriori subcell FV limiter. The setup of the test is the same as in the previous section, but on a three dimensional domain $\Omega=[-5,5] \mathrm{km} \times[-5,5] \mathrm{km} \times[-2.5,2.5] \mathrm{km}$ covered with $40 \times 40 \times 20$ elements on the coarsest mesh level plus one AMR refinement level and polynomial approximation degree $N=M=2$. We impose an initial load of $\sigma_{y y}=-120 \mathrm{MPa}$ and $\sigma_{x y}=70 \mathrm{MPa}$ and we pre-damage a zone of $33^{3} \mathrm{~m}^{3}$ in the origin characterized by $\xi=0.5$. The material properties are those associated to a weaker version of ROCK 1 , where $Y_{0}=1.75 \mathrm{GPa}$. The rupture propagation at times $t=[0.2,0.4,0.6,0.8]$ is shown in Fig. 18 and shows initially the formation of two planes aligned with the main eigenvectors of the initial stress. Then we can observe also in this test that some three-dimensional bifurcations start.

\subsection{Dynamic rupture in complex geometry}

As a last showcase, in this section we combine dynamic rupture as nonlinear coupling of fracture and wave propagation in complex geometry in $2 \mathrm{D}$ and $3 \mathrm{D}$. The computational domain is taken as $\Omega=[-8000,8000]^{2}$ and a level zero mesh with $25 \times 25$ elements is used, in combination with a polynomial approximation degree of $N=M=3$. Two AMR refinement levels are allowed. As initial condition we set a p-wave traveling in the $y$-direction, namely

$$
\begin{aligned}
\boldsymbol{\sigma} & =+\epsilon R_{\sigma} \cdot \exp \left(-\frac{1}{2} \frac{\left(x-x_{0}\right)^{2}}{\delta^{2}}\right) \\
v & =-\epsilon c_{p} \cdot \exp \left(-\frac{1}{2} \frac{\left(x-x_{0}\right)^{2}}{\delta^{2}}\right) \\
u & =w=0
\end{aligned}
$$
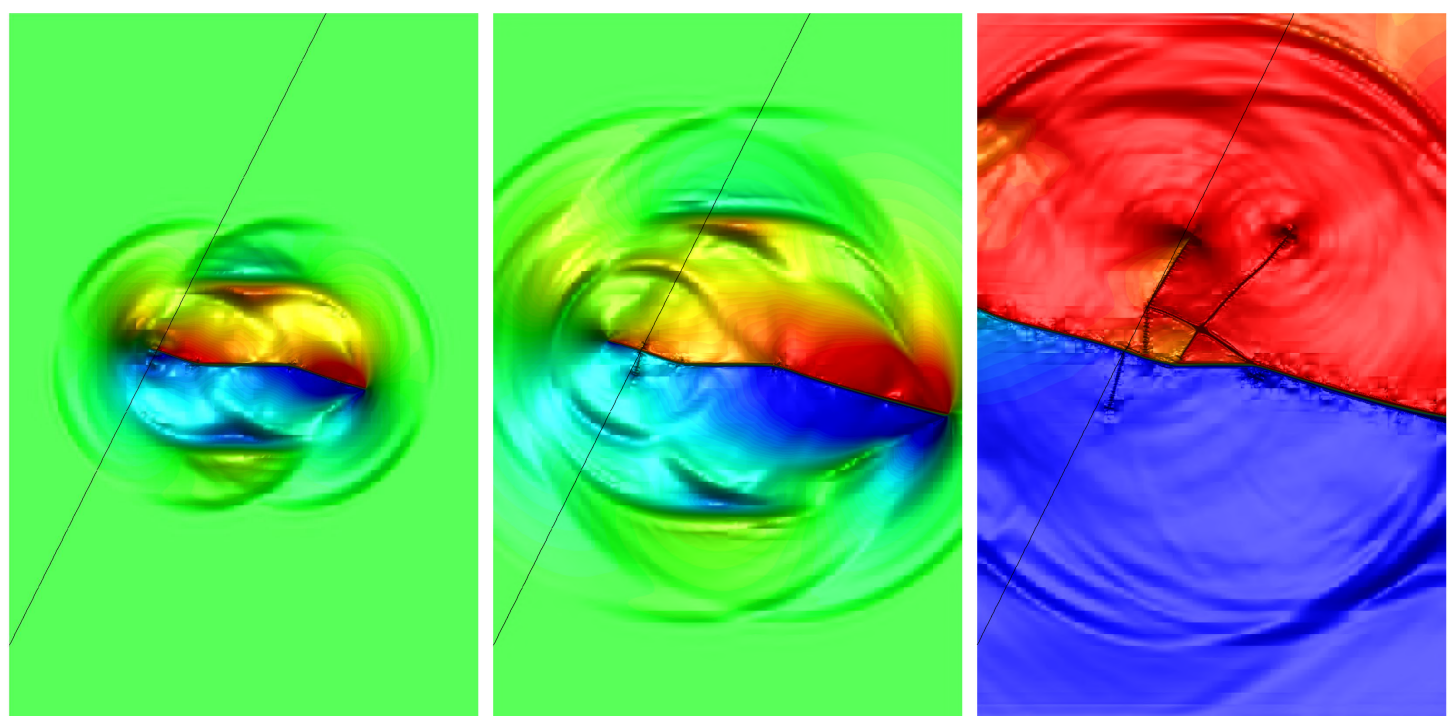

Figure 16: Evolution of the out of plane rupture in a heterogeneous material at times $t=[0.5,0.9,5.0]$. The separation line is reported and the two material are characterized by different critical stress $Y_{0}$, i.e. $Y_{0}=180 \mathrm{MPa}$ and $Y_{0}=240 \mathrm{MPa}$ respectively for the first and the second material. Super shear can be observed in the right crack propagation while crack tends naturally to propagate parallel to the separation line of the two materials 
where $R_{\sigma}=(\lambda, \lambda+2 \mu, \lambda, 0,0,0), x_{0}=-4000, \delta=1000.0$ and $\epsilon=1.79 \cdot 10^{8} / 2 \mu$. The material is assumed to be ROCK 1 , with an equivalent stress simply defined through the von Mises stress. The complex geometry of the free surface of the solid material is described by the following profile:

$$
y_{s}(x)=6000-500 \sin \left(10^{-3} x\right) .
$$

The diffuse interface function $\alpha$ is then assumed to be $\alpha=1$ below the surface $\left(y<y_{s}\right)$ and $\alpha=0$ above, i.e. $y>y_{s}$. The step function in $\alpha$ is then appropriately smoothed with a characteristic smoothing length of $d=300$, see [115] for details. Finally, we set $\xi=1$ in a point placed in $(x, y)=(3000,-2000)$ and on a line with inclination $\theta=\pi / 4$ and horizontal length of $2000 \sqrt{2}$. In Figure 19 we report the evolution of the traveling p-wave in terms of equivalent stress (right column) and horizontal velocity (left column). We note how the crack generates and propagates away from the pre-damaged point in a similar way as observed in section 4.5. On the damaged line we see the dislocation through velocity gradients and some bifurcation effects on the main crack line.

We now perform a similar test in three-space dimensions. Here the domain $\Omega=[-8000,8000]^{3}$ is covered with an initially regular Cartesian mesh of $25 \times 25 \times 25$ elements and polynomial ap-
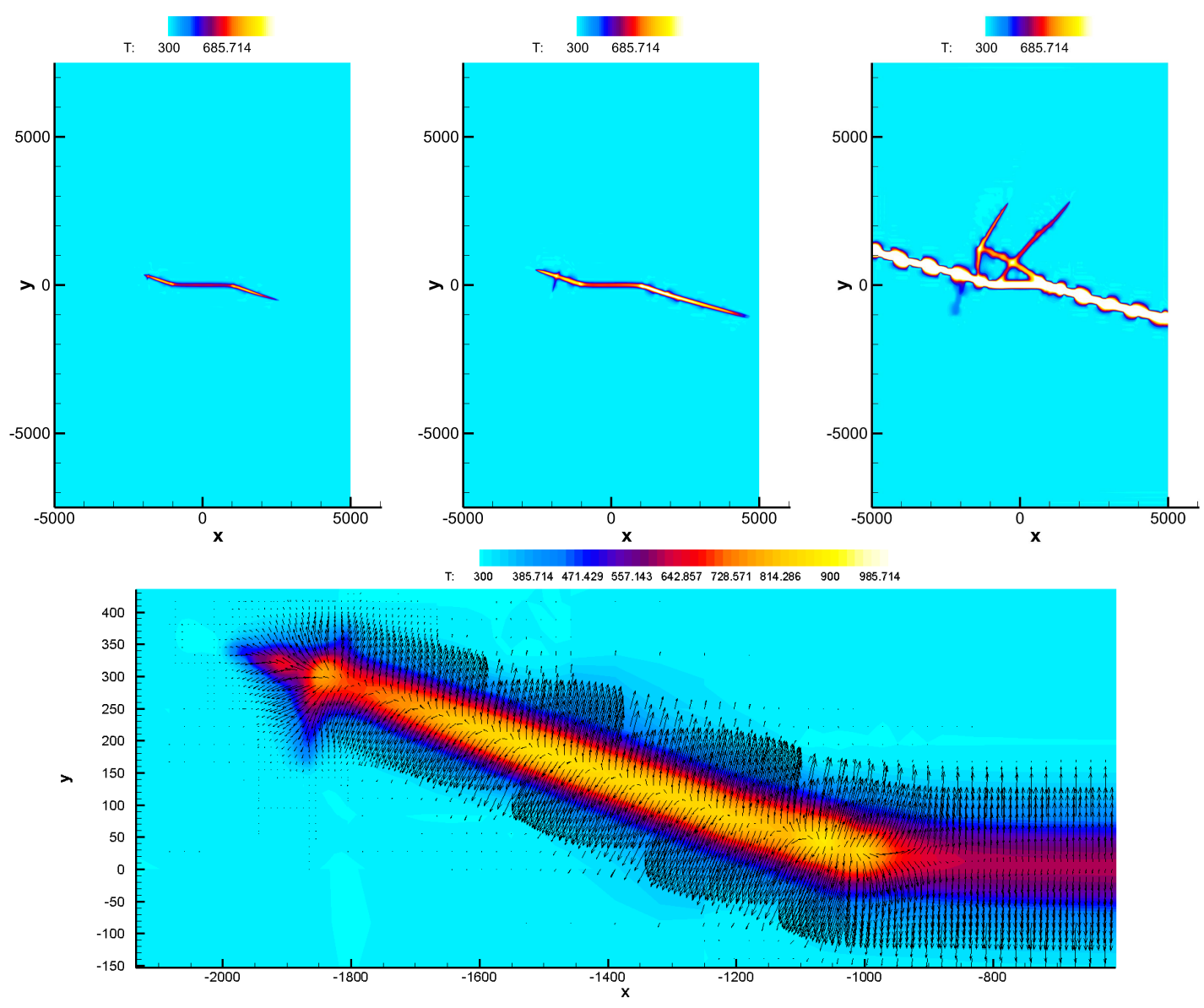

Figure 17: Evolution of the thermal trace generated by friction during the sliding process (top figures); representation of the heat flux vector $\left(J_{x}, J_{z}\right)$ at $t=0.9$ in the bottom figure, showing the heat propagation during the process. 
proximation degree $N=M=2$. Then, one level of AMR refinement is allowed. For the definition of the complex geometry we extract a zone of size $(16 \mathrm{~km})^{3}$ from real DTM data centered in UTM coordinate $\mathbf{x}=[4456.397222711,2596.544914552] \mathrm{km}$, which is within the Trentino-Südtirol region in Northern Italy. As material we consider a weaker version of ROCK 1 , where $Y_{0}=1.75 \mathrm{GPa}$. The pre-damaged fault line is then extended in the $z$ - direction for a total size of $2 \mathrm{~km}$. The time evolution of the p-wave, hitting first the pre-damaged zone and then the complex geometry is reported in Figure 20. The dynamic stresses transferred by the propagating waves cause complex crack propagation across the predefined weak fault zone. In Figure 21 we report the iso-lines of the horizontal velocity component $u$ on the complex geometry. We underline here that the $u$ component is generated due to the complex topology and underground structure. Finally we report the profile $\alpha=0.5$ at position $\{z \approx 4300\}$ before and after the impact in Figure 22, where we can observe a small displacement. As already observed in [115], this is possible because according to Eqn. (1a), the material parameters and the solid volume fraction function are free to move according to the local velocity field.
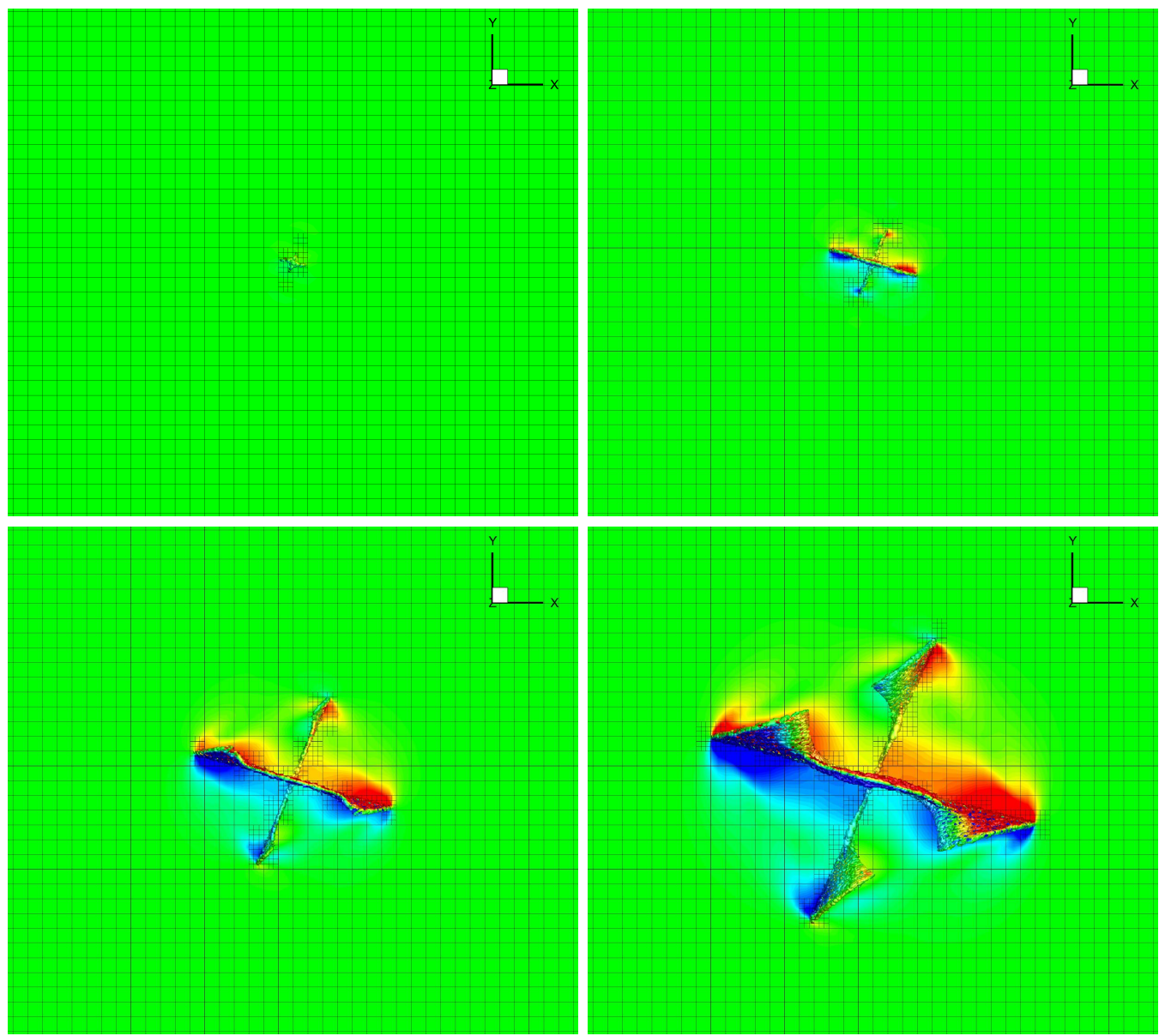

Figure 18: Resulting rupture front for the $3 D$ out of plane rupture colored using the horizontal velocity $u$. 

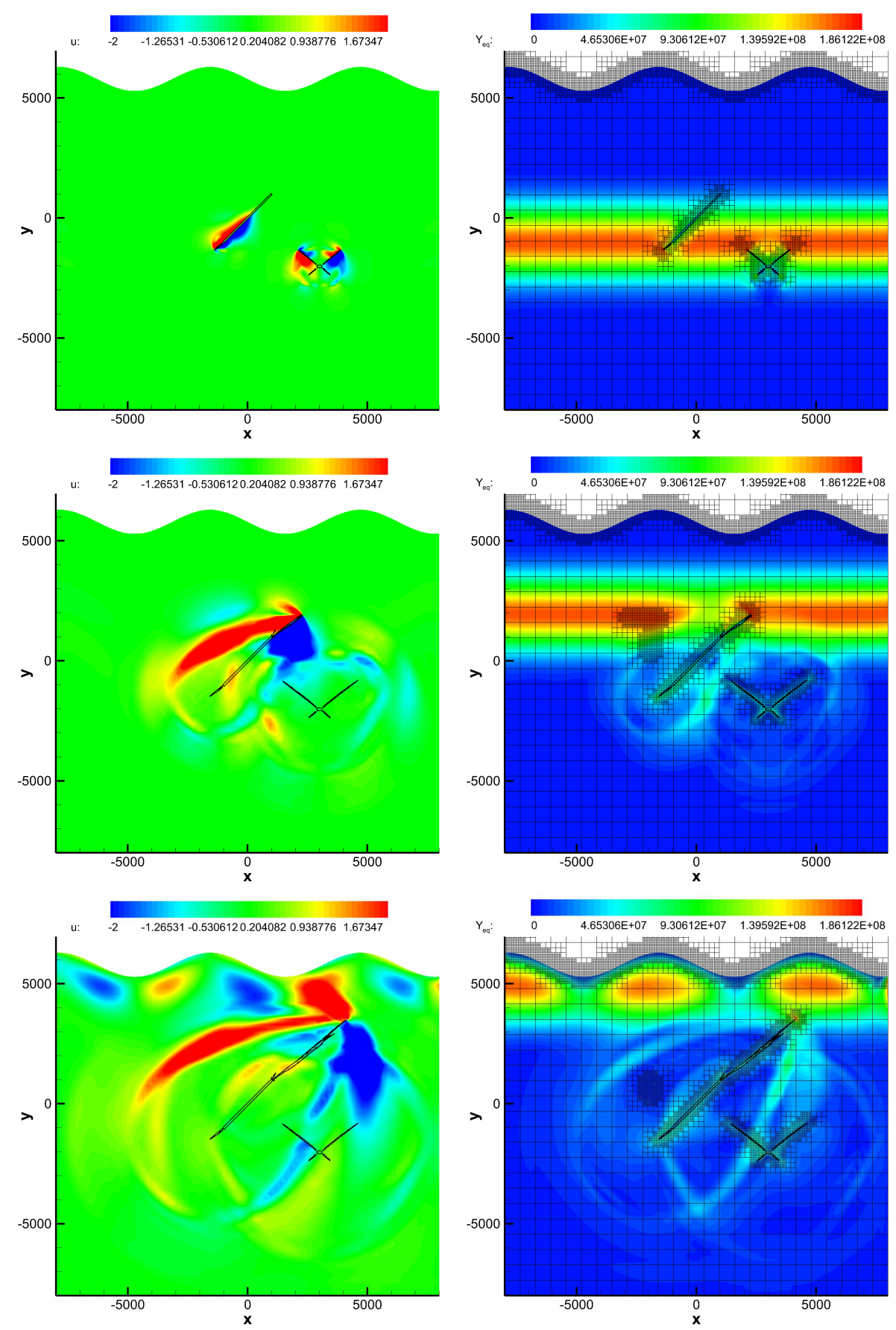

Figure 19: P-wave hitting a complex geometry with free crack generation at times, from top to bottom, $t=0.5,1.0$ and $1.5 \mathrm{~s}$. In the left column we report the velocity component $u$ that is only generated due to sliding and complex geometry, in the right column we report the equivalent stress value that depict the traveling p-wave and the interaction with the underground structure. 

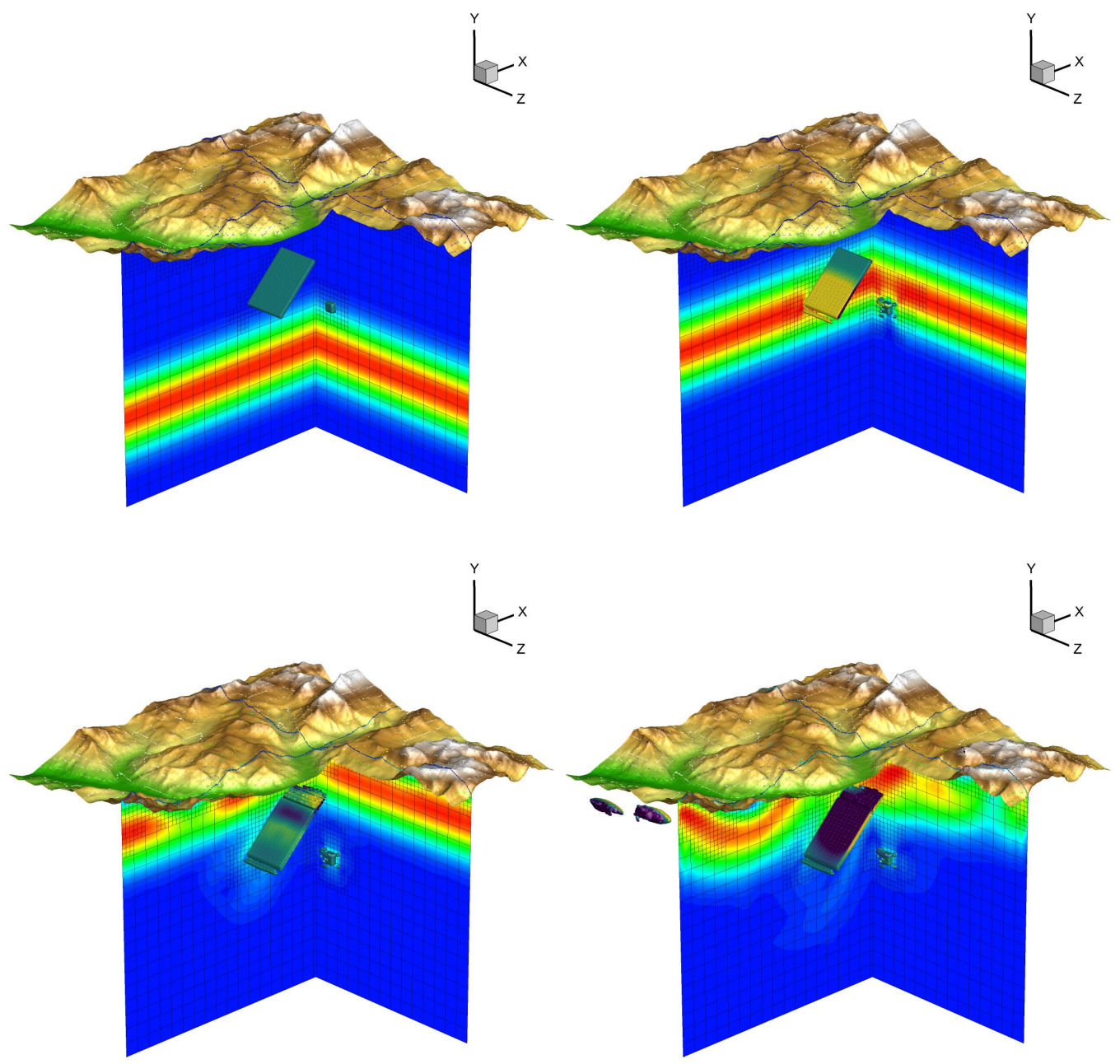

Figure 20: $\mathrm{P}$-wave propagating in a $3 D$ complex geometry, dynamically triggering spontaneous crack generation across a predefined weak fault zone at times $t=[0.0,0.5,1.0,1.4]$ from top left to bottom right. The mountain shape is obtained by extracting the isosurface $\alpha=0.5$, whose values are determined from a real DTM data of extent $16 \mathrm{~km}$ in each direction, centered in $\mathbf{x}=[4456.397222711,2596.544914552]$. 


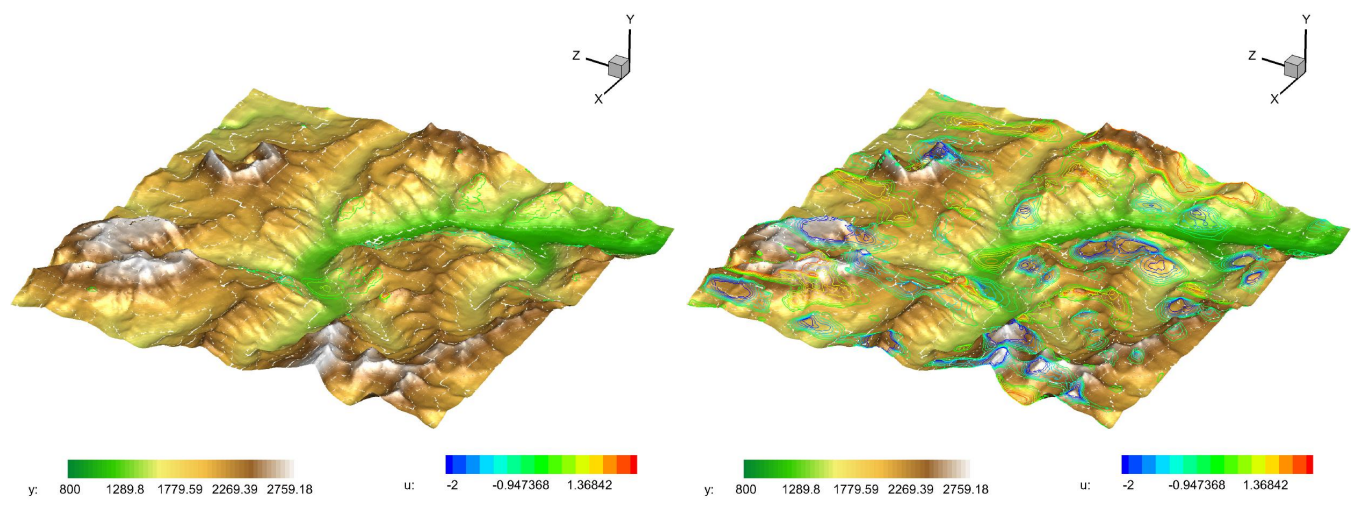

Figure 21: Velocity component $u$ computed on the surface at the beginning of the impact $t=1.0$ (left) and after the impact at $t=1.4$ (right). We underline that the component $u$ is generated only by sliding and complex geometry shape as for the $2 D$ case.

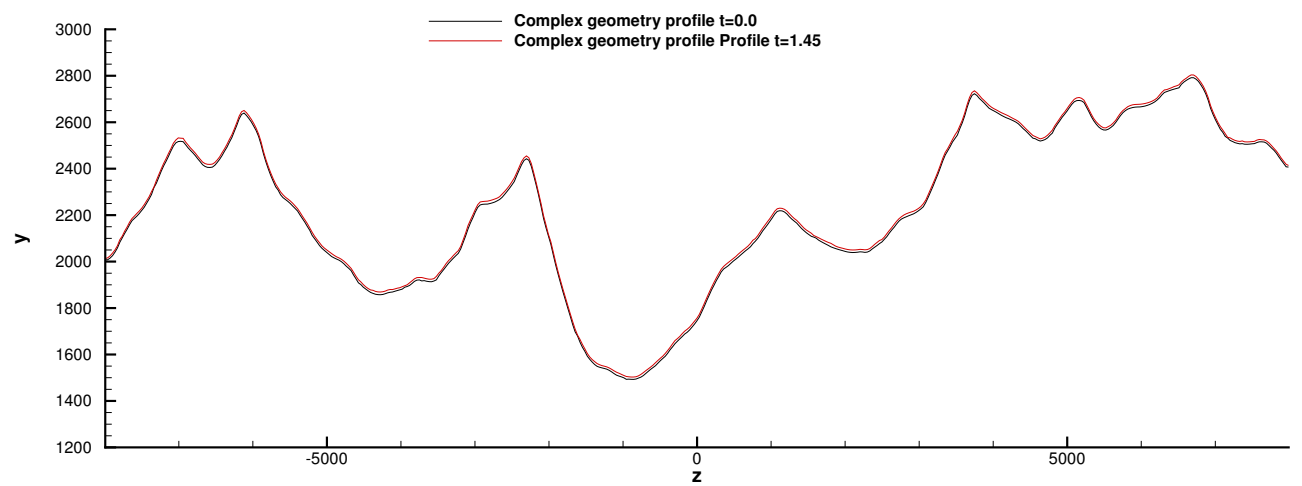

Figure 22: Elevation profile through the isoline $z \approx 4300$ extracted from the isosurface $\alpha=0.5$ at times $t=0.0$ and $t=1.4$ shows the displacement generated by the impact of the $\mathrm{p}$-wave with the surface due to the transport contribution in the equation for the color function $\alpha$. 


\section{Conclusion}

In this paper we have extended the Godunov-Peshkov-Romenski (GPR) model of continuum mechanics in Eulerian coordinates $[64,106,96,47]$ by an additional dynamic process that describes material failure. More precisely, the extension consists in the addition of a scalar material damage variable $\xi \in[0,1]$, where $\xi=0$ indicates the fully intact material and $\xi=1$ denotes the fully damaged material. The parameter is governed by an advection-reaction equation, with a highly nonlinear reaction source term that is to a certain extent similar to the reaction source terms that are used to describe reaction kinetics in chemistry. In the present model, the reaction source term depends essentially on the ratio of the equivalent stress (e.g. the von Mises stress) to the yield stress. The underlying ideas go back to previous work of Resnyansky et al. and Romenski [103, 107], but in the present paper the GPR model with material damage has for the first time solved with high order schemes on space-time adaptive AMR meshes and has furthermore been combined with the diffuse interface approach introduced in $[115,24]$, which allows an easy description of moving elastic bodies on space-time adaptive Cartesian meshes simply via the evolution of an additional scalar solid volume fraction function. The reaction source terms in the governing equation for the damage variable are extremely stiff, and can in general not be properly integrated in time via a simple implicit Euler method. For this purpose, in this paper we rely on simple operator splitting, combined with a very efficient and accurate exponential time integrator. The proposed time integrator has been carefully validated against standard software packages for stiff ODE.

Via numerical experiments we have shown that the proposed mathematical model is able to describe brittle material as well as ductile material if the model parameters are properly chosen. The model is also able to produce rate-dependent stress-strain diagrams, and also contains all the necessary ingredients to describe the phenomenon of material fatigue. To the best knowledge of the authors, this is the first time that fatigue has been studies in the context of the GPR model of continuum mechanics.

We have shown numerical results for some test problems with material failure, for which experimental reference data were available. Last but not least, we have also provided a first simplified proof of concept that the computational approach proposed in this paper can at least in principle be also used in the context of describing nonlinear seismic wave propagation and subsequent dynamic rupture processes in complex $3 \mathrm{D}$ geometries at a regional scale.

In the future we plan to use the methodology developed in this paper more extensively for the numerical simulation of nonlinear wave propagation and dynamic rupture processes in computational seismology. We also want to investigate in more detail the rheologies of broken brittle material, which should behave more like a granular fluid rather than an elastic solid. In this case, the correct asymptotic behaviour of the relaxation time $\tau_{1}$ and of the shear sound speed $c_{s}$ in the limit $\xi \rightarrow 1$ will be of fundamental importance. Further research on this topic will be carried out in the future.

\section{Acknowledgments}

The research presented in this paper has been financed by the European Union's Horizon 2020 Research and Innovation Programme under the project ExaHyPE, grant agreement number no. 671698 (call FETHPC-1-2014). Results by E.R. obtained in Sects. 2 and 4 were supported by the Russian Science Foundation grant (project 19-77-20004). S.C. acknowledges the financial support received by the Deutsche Forschungsgemeinschaft (DFG) under the project Droplet Interaction Technologies (DROPIT), grant no. GRK 2160/1. A.-A.G. acknowledges additional funding from the European Union's Horizon 2020 research and innovation programme under the projects TEAR, grant agreement No. 852992 (ERC-StG) and ChEESE, grant agreement No. 823844. M.D. also acknowledges the financial support received from the Italian Ministry of Education, University and 
Research (MIUR) in the frame of the Departments of Excellence Initiative 2018-2022 attributed to DICAM of the University of Trento (grant L. 232/2016) and in the frame of the PRIN 2017 project Innovative numerical methods for evolutionary partial differential equations and applications. M.D. has also received funding from the University of Trento via the Strategic Initiative Modeling and Simulation. M.D. is member of the GNCS-INdAM group.

\section{References}

[1] E. Abbate, A. Iollo, and G. Puppo. An asymptotic-preserving all-speed scheme for fluid dynamics and nonlinear elasticity. SIAM Journal on Scientific Computing, 41:A2850-A2879, 2019.

[2] R. Abgrall. How to prevent pressure oscillations in multicomponent flow calculations: A quasi conservative approach. Journal of Computational Physics, 125:150-160, 1996.

[3] R. Abgrall and S. Karni. A comment on the computation of non-conservative products. Journal of Computational Physics, 229:2759-2763, 2010.

[4] Awad H. Al-Mohy and Nicholas J. Higham. A new scaling and squaring algorithm for the matrix exponential. SIAM J. Matrix Anal. Appl., 31(3):970-989, 2009.

[5] Awad H. Al-Mohy and Nicholas J. Higham. Computing the action of the matrix exponential, with an application to exponential integrators. SIAM J. Scientific Computing, 33(2):488-511, 2011.

[6] M. Ambati, T. Gerasimov, and L. de Lorenzis. Phase-field modeling of ductile fracture. Comput. Mech., $55: 1017-1040,2015$.

[7] M. Ambati, T. Gerasimov, and L. de Lorenzis. A review on phase-field models of brittle fracture and a new fast hybrid formulation. Comput. Mech., 55:383-405, 2015.

[8] D.J. Andrews. Rupture dynamics with energy loss outside the slip zone. Journal of Geophysical Research: Solid Earth, 110(B1), 2005.

[9] D.S. Balsara, C. Meyer, M. Dumbser, H. Du, and Z. Xu. Efficient implementation of ADER schemes for Euler and magnetohydrodynamical flows on structured meshes - Speed comparisons with Runge-Kutta methods. Journal of Computational Physics, 235:934-969, 2013.

[10] P. Barton and E. Romenski. On computational modelling of strain-hardening material dynamics. Communications in Computational Physics, 11(5):1525-1546, 2012.

[11] P. T. Barton. An eulerian method for finite deformation anisotropic damage with application to high strainrate problems. International Journal of Plasticity, 83:225 - 251, 2016.

[12] P. T. Barton. An interface-capturing Godunov method for the simulation of compressible solid-fluid problems. Journal of Computational Physics, 390:25-50, 2019.

[13] P.T. Barton, D. Drikakis, E. Romenski, and V.A. Titarev. Exact and approximate solutions of Riemann problems in non-linear elasticity. Journal of Computational Physics, 228(18):7046-7068, 2009.

[14] P.T. Barton, D. Drikakis, and E.I. Romenski. An eulerian finite-volume scheme for large elastoplastic deformations in solids. International Journal for Numerical Methods in Engineering, 81(4):453-484, 2010.

[15] J. Bell, M. Berger, J. Saltzman, and M. Welcome. Three-dimensional adaptive mesh refinement for hyperbolic conservation laws. SIAM J. Sci. Comput., 15(1):127-138, January 1994.

[16] M. J. Berger and P. Colella. Local adaptive mesh refinement for shock hydrodynamics. Journal of Computational Physics, 82:64-84, May 1989.

[17] M. J. Berger and A. Jameson. Automatic adaptive grid refinement for the Euler equations. AIAA Journal, 23:561-568, 1985 .

[18] M. J. Berger and R. LeVeque. Adaptive mesh refinement using wave-propagation algorithms for hyperbolic systems. SIAM Journal on Numerical Analysis, 35:2298-2316, December 1998.

[19] M. J. Berger and J. Oliger. Adaptive Mesh Refinement for Hyperbolic Partial Differential Equations. Journal of Computational Physics, 53:484, March 1984

[20] Harsha S Bhat, Ares J Rosakis, and Charles G Sammis. A micromechanics based constitutive model for brittle failure at high strain rates. Journal of Applied Mechanics, 79(3), 2012.

[21] N. Bourne, L.C.Forde, and J.E. Field. High-speed photography and stress-gauge studies of the impact and penetration of plates by rods. Speed Photography and Photonics, 2869:626, 1997.

[22] N. Bourne, Z. Rosenberg, Y. Mebar, T. Obara, and J. Field. A high-speed photographic study of fracture wave propagation in glasses. Journal de Physique, IV:C8-635-640, 1994.

[23] H.J. Bungartz, M. Mehl, T. Neckel, and T. Weinzierl. The PDE framework Peano applied to fluid dynamics: An efficient implementation of a parallel multiscale fluid dynamics solver on octree-like adaptive Cartesian grids. Computational Mechanics, 46:103-114, 2010.

[24] S. Busto, S. Chiocchetti, M. Dumbser, E. Gaburro, and I. Peshkov. High order ADER schemes for continuum mechanics. Frontiers in Physics, 8:32, 2020.

[25] V. Carollo, T. Guillén-Hernández, J. Reinoso, and M. Paggi. Recent advancements on the phase field approach to brittle fracture for heterogeneous materials and structures. Adv. Model. and Simul. in Eng. Sci., 5(8), 2018. 
[26] M.J. Castro, J.M. Gallardo, and A. Marquina. Approximate Osher-Solomon schemes for hyperbolic systems. Applied Mathematics and Computation, 272:347-368, 2016.

27] M.J. Castro, J.M. Gallardo, and C. Parés. High-order finite volume schemes based on reconstruction of states for solving hyperbolic systems with nonconservative products. applications to shallow-water systems. Mathematics of Computation, 75:1103-1134, 2006.

[28] M.J. Castro, P.G. LeFloch, M.L. Muñoz-Ruiz, and C. Parés. Why many theories of shock waves are necessary: Convergence error in formally path-consistent schemes. Journal of Computational Physics, 227:8107-8129, 2008.

[29] S. Clain, S. Diot, and R. Loubère. A high-order finite volume method for systems of conservation lawsâĂŤmulti-dimensional optimal order detection (MOOD). Journal of Computational Physics, 230(10):4028 $-4050,2011$.

[30] C.Miehe, S. Teichtmeister, and F. Aldakheel F. Phase-field modelling of ductile fracture: a variational gradient-extended plasticity-damage theory and its micromorphic regularization. Phil. Trans. R. Soc. A, (37420150170), 2016.

[31] B. Cockburn, S. Hou, and C. W. Shu. The Runge-Kutta local projection discontinuous Galerkin finite element method for conservation laws IV: the multidimensional case. Mathematics of Computation, 54:545-581, 1990.

32] B. Cockburn, S. Y. Lin, and C.W. Shu. TVB Runge-Kutta local projection discontinuous Galerkin finite element method for conservation laws III: one dimensional systems. Journal of Computational Physics, 84:90-113, 1989.

[33] B. Cockburn and C. W. Shu. TVB Runge-Kutta local projection discontinuous Galerkin finite element method for conservation laws II: general framework. Mathematics of Computation, 52:411-435, 1989.

[34] B. Cockburn and C. W. Shu. The Runge-Kutta discontinuous Galerkin method for conservation laws V: multidimensional systems. Journal of Computational Physics, 141:199-224, 1998.

[35] L. A. Dalguer, K. Irikura, and J. D. Riera. Simulation of tensile crack generation by threeâĂ dynamic shear rupture propagation during an earthquake. Journal of Geophysical Research: Solid Earth, 108:2144, 2003.

[36] Alexia de Brauer, Angelo Iollo, and Thomas Milcent. A Cartesian Scheme for Compressible Multimaterial Hyperelastic Models with Plasticity. Communications in Computational Physics, 22(05):1362-1384, 2017.

[37] S. Diot, S. Clain, and R. Loubère. Improved detection criteria for the multi-dimensional optimal order detection (MOOD) on unstructured meshes with very high-order polynomials. Computers and Fluids, 64:43 $-63,2012$.

[38] S. Diot, R. Loubère, and S. Clain. The MOOD method in the three-dimensional case: Very-high-order finite volume method for hyperbolic systems. International Journal of Numerical Methods in Fluids, 73:362-392, 2013.

[39] M. Dumbser, D. Balsara, E.F. Toro, and C.D. Munz. A unified framework for the construction of one-step finite-volume and discontinuous Galerkin schemes. Journal of Computational Physics, 227:8209-8253, 2008.

[40] M. Dumbser and D.S. Balsara. A new, efficient formulation of the HLLEM Riemann solver for general conservative and non-conservative hyperbolic systems. Journal of Computational Physics, 304:275-319, 2016.

[41] M. Dumbser, M. Castro, C. Parés, and E.F. Toro. ADER schemes on unstructured meshes for non-conservative hyperbolic systems: Applications to geophysical flows. Computers and Fluids, 38:1731-âĂŞ1748, 2009.

[42] M. Dumbser, C. Enaux, and E.F. Toro. Finite volume schemes of very high order of accuracy for stiff hyperbolic balance laws. Journal of Computational Physics, 227:3971-4001, 2008.

[43] M. Dumbser, A. Hidalgo, M. Castro, C. Parés, and E.F. Toro. FORCE schemes on unstructured meshes II: Non-conservative hyperbolic systems. Computer Methods in Applied Mechanics and Engineering, 199:625647, 2010.

[44] M. Dumbser and M. Käser. Arbitrary high order non-oscillatory finite volume schemes on unstructured meshes for linear hyperbolic systems. Journal of Computational Physics, 221:693-723, 2007.

[45] M. Dumbser, M. Käser, V.A Titarev, and E.F. Toro. Quadrature-free non-oscillatory finite volume schemes on unstructured meshes for nonlinear hyperbolic systems. Journal of Computational Physics, 226:204-243, 2007.

[46] M. Dumbser and C.D. Munz. Building blocks for arbitrary high order discontinuous Galerkin schemes. Journal of Scientific Computing, 27:215-230, 2006.

[47] M. Dumbser, I. Peshkov, E. Romenski, and O. Zanotti. High order ader schemes for a unified first order hyperbolic formulation of continuum mechanics: Viscous heat-conducting fluids and elastic solids. Journal of Computational Physics, 314:824-862, 2016.

[48] M. Dumbser and E. F. Toro. On universal Osher-type schemes for general nonlinear hyperbolic conservation laws. Communications in Computational Physics, 10:635-671, 2011.

[49] M. Dumbser and E. F. Toro. A simple extension of the Osher Riemann solver to non-conservative hyperbolic systems. Journal of Scientific Computing, 48:70-88, 2011.

[50] M. Dumbser and O. Zanotti. Very high order PNPM schemes on unstructured meshes for the resistive relativistic MHD equations. Journal of Computational Physics, 228:6991-7006, 2009.

[51] M. Dumbser, O. Zanotti, A. Hidalgo, and D.S. Balsara. ADER-WENO Finite Volume Schemes with Space- 
Time Adaptive Mesh Refinement. Journal of Computational Physics, 248:257-286, 2013.

[52] M. Dumbser, O. Zanotti, R. Loubère, and S. Diot. A posteriori subcell limiting of the discontinuous Galerkin finite element method for hyperbolic conservation laws. Journal of Computational Physics, 278:47-75, December 2014

[53] B. Einfeldt. On Godunov-type methods for gas dynamics. SIAM J. Numer. Anal., 25:294-318, 1988.

[54] B. Einfeldt, C. D. Munz, P. L. Roe, and B. Sjögreen. On Godunov-type methods near low densities. Journal of Computational Physics, 92:273-295, 1991.

[55] N. Favrie and S.L. Gavrilyuk. Diffuse interface model for compressible fluid-compressible elastic-plastic solid interaction. Journal of Computational Physics, 231:2695âĂŞ2723, 2012.

[56] N. Favrie, S.L. Gavrilyuk, and R.Saurel. Solid-fluid diffuse interface model in cases of extreme deformations. Journal of Computational Physics, 228:6037-6077, 2009.

[57] G.A. Francfort and J.-J. Marigo. Revisiting brittle fracture as an energy minimization problem. Journal of the Mechanics and Physics of Solids, 46(8):1319-1342, 1998.

[58] A.-A. Gabriel, J.P. Ampuero, L.A. Dalguer, and P.M. Mai. Source properties of dynamic rupture pulses with off-fault plasticity. Journal of Geophysical Research: Solid Earth, 118:4117-4126, 2013.

[59] G. Gassner, M. Dumbser, F. Hindenlang, and C.D. Munz. Explicit one-step time discretizations for discontinuous Galerkin and finite volume schemes based on local predictors. Journal of Computational Physics, 230:4232-4247, 2011.

[60] S.L. Gavrilyuk, N. Favrie, and R. Saurel. Modelling wave dynamics of compressible elastic materials. Journal of Computational Physics, 227(5):2941-2969, feb 2008.

[61] S.K. Godunov. Finite difference methods for the computation of discontinuous solutions of the equations of fluid dynamics. Mathematics of the USSR - Sbornik, 47:271-306, 1959.

[62] S.K. Godunov. An interesting class of quasilinear systems. Dokl. Akad. Nauk SSSR, 139(3):521-523, 1961.

[63] SK Godunov. Symmetric form of the magnetohydrodynamic equation. Numerical Methods for Mechanics of Continuum Medium, 3(1):26-34, 1972.

64] S.K. Godunov and E.I. Romenski. Nonstationary equations of nonlinear elasticity theory in eulerian coordinates. Journal of Applied Mechanics and Technical Physics, 13(6):868-884, 1972.

[65] S.K. Godunov and E.I. Romenski. Nonstationary equations of the nonlinear theory of elasticity in Euler coordinates. Journal of Applied Mechanics and Technical Physics, 13:868-885, 1972.

[66] S.K. Godunov and E.I. Romenski. Thermodynamics, conservation laws and symmetric forms of differential equations in mechanics of continuous media. Computational fluid dynamics review, 95:19-31, 1995.

[67] S.K. Godunov and E.I. Romenski. Elements of Continuum Mechanics and Conservation Laws. Kluwer Academic/Plenum Publishers, 2003.

[68] H. Haeri, K. Shahriar, M. F. Marji, and P. Moarefvand. Experimental and numerical study of crack propagation and coalescence in pre-cracked rock-like disks. International Journal of Rock Mechanics and Mining Sciences, 67:20-28, 2014.

[69] A. Harten, B. Engquist, S. Osher, and S. Chakravarthy. Uniformly high order essentially non-oscillatory schemes, III. Journal of Computational Physics, 71:231-303, 1987.

[70] A. Harten, P.D. Lax, and B. van Leer. On upstream differencing and godunov-type schemes for hyperbolic conservation laws. SIAM Review, 25(1):35-61, 1983.

[71] A. Hidalgo and M. Dumbser. ADER schemes for nonlinear systems of stiff advection-diffusion-reaction equations. Journal of Scientific Computing, 48:173-189, 2011.

[72] Nicholas J. Higham. The scaling and squaring method for the matrix exponential revisited. SIAM J. Matrix Anal. Appl., 26(4):1179-1193, April 2005.

[73] Alan C. Hindmarsh. Lsode and lsodi, two new initial value ordinary differential equation solvers. SIGNUM Newsl., 15(4):10âĂŞ11, December 1980.

[74] Alan C. Hindmarsh. ODEPACK, A Systematized Collection of ODE Solvers, pages 55-64. North-Holland, Amsterdam, 1983.

[75] H. Jackson. On the eigenvalues of the ader-weno galerkin predictor. Journal of Computational Physics, 333:409-413, 2017.

[76] H. Jackson and N. Nikiforakis. A numerical scheme for non-Newtonian fluids and plastic solids under the GPR model. Journal of Computational Physics, 387:410-429, 2019.

[77] H. Jackson and N. Nikiforakis. A unified Eulerian framework for multimaterial continuum mechanics. Journal of Computational Physics, 401:109022, 2019.

[78] M. Käser and M. Dumbser. An arbitrary high order discontinuous Galerkin method for elastic waves on unstructured meshes I: The two-dimensional isotropic case with external source terms. Geophysical Journal International, 166:855-877, 2006.

79] M. Kikuchi. Inelastic effects on crack propagation. Journal of Physics of the Earth, 23(2):161-172, 1975.

[80] R. J. LeVeque. Finite Volume Methods for Hyperbolic Problems. Cambridge University Press, 2002.

[81] R. Loubère, M. Dumbser, and S. Diot. A new family of high order unstructured MOOD and ADER finite volume schemes for multidimensional systems of hyperbolic conservation laws. Communication in Computational Physics, 16:718-763, 2014 
[82] H. Luo, L. Luo, R. Nourgaliev, V.A. Mousseau, and N. Dinh. A reconstructed discontinuous Galerkin method for the compressible NavierâĂŞStokes equations on arbitrary grids. Journal of Computational Physics, 229:6961-6978, 2010.

[83] H. Luo, Y. Xia, S. Spiegel, R. Nourgaliev, and Z. Jiang. A reconstructed discontinuous Galerkin method based on a Hierarchical WENO reconstruction for compressible flows on tetrahedral grids. Journal of Computational Physics, 236:477-492, 2013.

[84] Vladimir Lyakhovsky, Yehuda Ben-Zion, and Amotz Agnon. A viscoelastic damage rheology and rate-and state-dependent friction. Geophysical Journal International, 161(1):179-190, 2005.

[85] G. Dal Maso, P.G. LeFloch, and F. Murat. Definition and weak stability of nonconservative products. $J$. Math. Pures Appl., 74:483-548, 1995.

[86] A. Meister and S. Ortleb. A positivity preserving and well-balanced DG scheme using finite volume subcells in almost dry regions. Applied Mathematics and Computation, 272:259-273, 2016.

[87] N. Moës, J. Dolbow, and T. Belytschko. A finite element method for crack growth without remeshing. International Journal for Numerical Methods in Engineering, 46(1):131-150, 1999.

[88] L.O. Müller, C. Parés, and E.F. Toro. Well-balanced high-order numerical schemes for one-dimensional blood flow in vessels with varying mechanical properties. Journal of Computational Physics, 242:53-85, 2013.

[89] L.O. Müller and E.F. Toro. Well-balanced high-order solver for blood flow in networks of vessels with variable properties. International Journal for Numerical Methods in Biomedical Engineering, 29(12):1388-1411, 2013.

[90] S. Ndanou, N. Favrie, and S. Gavrilyuk. Criterion of hyperbolicity in hyperelasticity in the case of the stored energy in separable form. Journal of Elasticity, 115(1):1-25, 2014.

[91] S. Ndanou, N. Favrie, and S. Gavrilyuk. Multi-solid and multi-fluid diffuse interface model: Applications to dynamic fracture and fragmentation. Journal of Computational Physics, 295:523-555, 2015.

[92] Kurama Okubo, Harsha S Bhat, Esteban Rougier, Samson Marty, Alexandre Schubnel, Zhou Lei, Earl E Knight, and Yann Klinger. Dynamics, radiation, and overall energy budget of earthquake rupture with coseismic off-fault damage. Journal of Geophysical Research: Solid Earth, 124:11771-11801, 2019.

93] Kurama Okubo, Esteban Rougier, Zhou Lei, and Harsha S. Bhat. Modeling earthquakes with off-fault damage using the combined finite-discrete element method. Computational Particle Mechanics, 2020.

[94] C. Parés. Numerical methods for nonconservative hyperbolic systems: a theoretical framework. SIAM Journal on Numerical Analysis, 44:300-321, 2006.

[95] I. Peshkov, W. Boscheri, R. Loubère, E. Romenski, and M. Dumbser. Theoretical and numerical comparison of hyperelastic and hypoelastic formulations for Eulerian non-linear elastoplasticity. Journal of Computational Physics, 387:481-521, 2019.

[96] I. Peshkov and E. Romenski. A hyperbolic model for viscous newtonian flows. Continuum Mechanics and Thermodynamics, 28:85-104, 2016.

97] Ilya Peshkov, Michal Pavelka, Evgeniy Romenski, and Miroslav Grmela. Continuum Mechanics and Thermodynamics in the Hamilton and the Godunov-type Formulations. Continuum Mechanics and Thermodynamics, 2018.

[98] V. Peshkov. Second sound in helium ii. Journal of Physics (USSR), 8:381, 1944.

[99] R.M. Pidatella, G. Puppo, G. Russo, and P. Santagati. Semi-conservative finite volume schemes for conservation laws. SIAM Journal on Scientific Computing, 41:B576-B600, 2019.

[100] J. Qiu, M. Dumbser, and C.W. Shu. The discontinuous Galerkin method with Lax-Wendroff type time discretizations. Computer Methods in Applied Mechanics and Engineering, 194:4528-4543, 2005.

[101] A. Reinarz et al. Exahype: An engine for parallel dynamically adaptive simulations of wave problems. Computer Physics Communications, 254:107251, 2020.

[102] A. D. Resnyansky. Cth implementation of a two-phase material model with damage. Technical Report DSTO report DSTO-TR-2299, Defence Science and Technology Organisation, Edinburgh, Australia, 2009.

[103] A. D. Resnyansky, E. I. Romensky, and N. K. Bourne. Constitutive modeling of fracture waves. Journal of Applied Physics, 93(3):1537-1545, 2003.

[104] S. Rhebergen, O. Bokhove, and J.J.W. van der Vegt. Discontinuous Galerkin finite element methods for hyperbolic nonconservative partial differential equations. Journal of Computational Physics, 227:1887-1922, 2008.

[105] P.L. Roe. Approximate Riemann solvers, parameter vectors, and difference schemes. Journal of Computational Physics, 43:357-372, 1981

[106] E.I. Romenski. Hyperbolic systems of thermodynamically compatible conservation laws in continuum mechanics. Mathematical and computer modelling, 28(10):115-130, 1998.

[107] E. I. Romenskii. Deformation model for brittle materials and the structure of failure waves. Journal of Applied Mechanics and Technical Physics, 48(3):437-444, 2007.

[108] V. V. Rusanov. Calculation of interaction of non-steady shock waves with obstacles. J. Comput. Math. Phys. USSR, 1:267-279, 1961.

[109] T. Schwartzkopff, C.D. Munz, and E.F. Toro. ADER: A high order approach for linear hyperbolic systems in 2d. Journal of Scientific Computing, 17(1-4):231-240, 2002

[110] M. Sonntag and C.D. Munz. Shock capturing for discontinuous galerkin methods using finite volume subcells. 
In J. Fuhrmann, M. Ohlberger, and C. Rohde, editors, Finite Volumes for Complex Applications VII, pages 945-953. Springer, 2014.

[111] M. Sonntag and C.D. Munz. Efficient parallelization of a shock capturing for discontinuous Galerkin methods using finite volume sub-cells. Journal of Scientific Computing, 70:1262-1289, 2017.

[112] A. Spetz, R. Denzer, E. Tudisco, and O. Dahlblom. Phase-field fracture modelling of crack nucleation and propagation in porous rock. Int. J. Fract., 224:31-46, 2020

[113] A.H. Stroud. Approximate Calculation of Multiple Integrals. Prentice-Hall Inc., Englewood Cliffs, New Jersey, 1971.

[114] A. Taube, M. Dumbser, D. Balsara, and C.D. Munz. Arbitrary high order discontinuous Galerkin schemes for the magnetohydrodynamic equations. Journal of Scientific Computing, 30:441-464, 2007.

[115] M. Tavelli, M. Dumbser, D.E. Charrier, L. Rannabauer, T. Weinzierl, and M. Bader. A simple diffuse interface approach on adaptive cartesian grids for the linear elastic wave equations with complex topography. Journal of Computational Physics, 386:158-189, 2019.

[116] Marion Y Thomas and Harsha S Bhat. Dynamic evolution of off-fault medium during an earthquake: a micromechanics based model. Geophysical Journal International, 214(2):1267-1280, 2018

[117] M.Y. Thomas, H.S. Bhat, and Y. Klinger. Effect of Brittle Off-Fault Damage on Earthquake Rupture Dynamics, chapter 14, pages 255-280. American Geophysical Union (AGU), 2017.

[118] V.A. Titarev, E.I. Romenski, and E.F. Toro. MUSTA-type upwind fluxes for non-linear elasticity. International Journal for Numerical Methods in Engineering, 73:897-926, 2008.

[119] V.A. Titarev and E.F. Toro. ADER: Arbitrary high order Godunov approach. Journal of Scientific Computing, 17(1-4):609-618, December 2002

[120] V.A. Titarev and E.F. Toro. ADER schemes for three-dimensional nonlinear hyperbolic systems. Journal of Computational Physics, 204:715-736, 2005.

[121] E.F. Toro. Anomalies of conservative methods: analysis, numerical evidence and possible cures. Comput. Fluid Dyn. J., 11:128-143, 2002.

[122] E.F. Toro. Riemann Solvers and Numerical Methods for Fluid Dynamics. Springer, third edition, 2009.

[123] E.F. Toro, M. Spruce, and W. Speares. Restoration of the contact surface in the Harten-Lax-van Leer Riemann solver. Journal of Shock Waves, 4:25-34, 1994.

[124] E.F. Toro and V. A. Titarev. Solution of the generalized Riemann problem for advection-reaction equations. Proc. Roy. Soc. London, pages 271-281, 2002.

[125] M. Dumbser W. Boscheri and R. Loubere. Cell centered direct Arbitrary-Lagrangian-Eulerian ADER-WENO finite volume schemes for nonlinear hyperelasticity. Computers and Fluids, 134-135:111-129, 2016.

[126] T. Weinzierl and M. Mehl. Peano - A traversal and storage scheme for octree-like adaptive Cartesian multiscale grids. SIAM Journal on Scientific Computing, 33:2732-2760, 2011.

[127] P. Woodward and P. Colella. The numerical simulation of two-dimensional fluid flow with strong shocks. Journal of Computational Physics, 54:115-173, 1984.

[128] T. Yamashita. Generation of microcracks by dynamic shear rupture and its effects on rupture growth and elastic wave radiation. Geophysical Journal International, 143(2):395-406, 2000.

[129] O. Zanotti and M. Dumbser. A high order special relativistic hydrodynamic and magnetohydrodynamic code with space-time adaptive mesh refinement. Computer Physics Communications, 188:110-127, 2015.

[130] O. Zanotti and M. Dumbser. Efficient conservative ADER schemes based on WENO reconstruction and space-time predictor in primitive variables. Computational Astrophysics and Cosmology, 3:1-32, 2016

[131] O. Zanotti, F. Fambri, and M. Dumbser. Solving the relativistic magnetohydrodynamics equations with ADER discontinuous Galerkin methods, a posteriori subcell limiting and adaptive mesh refinement. Monthly Notices of the Royal Astronomical Society (MNRAS), 452:3010-3029, 2015.

[132] O. Zanotti, F. Fambri, M. Dumbser, and A. Hidalgo. Space-time adaptive ADER discontinuous Galerkin finite element schemes with a posteriori subcell finite volume limiting. Computers and Fluids, 118:204-224, 2015 . 


\section{Appendix A. Initialization of the distortion field $A$ from a given stress tensor}

A peculiar feature of the GPR model is that we evolve the distortion field $\mathbf{A}$ instead of the stress tensor $\boldsymbol{\sigma}$, or the strain tensor $\boldsymbol{\epsilon}$. The symmetric stress tensor (6 independent components) can be easily computed from $\mathbf{A}$ (9 independent components) using the definition $\sigma_{i k}=-\rho A_{j i} E_{A_{j k}}$. For the opposite direction $\boldsymbol{\sigma} \rightarrow \mathbf{A}$, we first need to remove the rotational degree of freedom contained in $\mathbf{A}$. In order to do that we write the matrix $\mathbf{A}$ as $\mathbf{A}=\mathbf{L}_{d} \cdot \mathbf{R}(\theta)$ where

$$
\mathbf{R}=\mathbf{R}(\theta)=\left(\begin{array}{ccc}
\cos (\theta) & -\sin (\theta) & 0 \\
\sin (\theta) & \cos (\theta) & 0 \\
0 & 0 & 1
\end{array}\right), \quad \mathbf{L}_{d}=\left(\begin{array}{ccc}
s_{1} & 0 & 0 \\
s_{2} & s_{3} & 0 \\
s_{4} & s_{5} & s_{6}
\end{array}\right)
$$

is a rotation matrix and a lower triangular matrix, respectively; $\theta$ is a chosen as the main direction. Now taking $E$ as in Eq. (3)-(4), $\boldsymbol{\sigma}$ and $p$ are computed according to their definition and then our target is that the quantity

$$
f(s)=\left(\begin{array}{c}
\sigma_{11}-p \\
\sigma_{22}-p \\
\sigma_{33}-p \\
\sigma_{12} \\
\sigma_{23} \\
\sigma_{13}
\end{array}\right)
$$

approaches our initial stress field $\Sigma^{0}=\left(\sigma_{11}^{0}, \sigma_{22}^{0}, \sigma_{33}^{0}, \sigma_{12}^{0}, \sigma_{23}^{0}, \sigma_{13}^{0}\right)$. In order to do that, we employ a simple Newton algorithm, that requires the computation of the Jacobian matrix

$$
J_{i j}=\frac{\partial f_{i}}{\partial s_{j}} \quad \forall i, j \in[1, \ldots, 6] .
$$

The explicit expression of the Jacobian for any generic angle $\theta$ can be cumbersome, but can be done at the aid of modern computer algebra systems. The algorithm is then defined as follows: start from an initial guess vector $\mathbf{s}^{0}=\left(s_{1}^{0} \ldots s_{6}^{0}\right)$, then $s^{k+1}=s^{k}-J^{-1} F\left(s^{k}\right)$ up to convergence of $F(s)=f(s)-\Sigma^{0}=0$. 